\title{
Spin $\frac{1}{2}^{+}$, spin $\frac{3}{2}^{+}$and transition magnetic moments of low lying and charmed baryons
}

\author{
Neetika Sharma $^{a}$, Harleen Dahiya ${ }^{a}$, P.K. Chatley ${ }^{a}$ and Manmohan Gupta ${ }^{b}$ \\ ${ }^{a}$ Department of Physics, Dr. B.R. Ambedkar National Institute of Technology, Jalandhar, 144011, India \\ ${ }^{b}$ Department of Physics, Centre of Advanced Study in Physics, Panjab University, Chandigarh 160014, India
}

(Dated: October 23, 2018)

\begin{abstract}
Magnetic moments of the low lying and charmed spin $\frac{1}{2}^{+}$and spin $\frac{3}{2}^{+}$baryons have been calculated in the $\mathrm{SU}(4)$ chiral constituent quark model $(\chi \mathrm{CQM})$ by including the contribution from $c \bar{c}$ fluctuations. Explicit calculations have been carried out for the contribution coming from the valence quarks, "quark sea" polarizations and their orbital angular momentum. The implications of such a model have also been studied for magnetic moments of the low lying spin $\frac{3}{2}^{+} \rightarrow \frac{1}{2}^{+}$ and $\frac{1}{2}^{+} \rightarrow \frac{1}{2}^{+}$transitions as well as the transitions involving charmed baryons. The predictions of $\chi \mathrm{CQM}$ not only give a satisfactory fit for the baryons where experimental data is available but also show improvement over the other models. In particular, for the case of $\mu(p), \mu\left(\Sigma^{+}\right), \mu\left(\Xi^{0}\right)$, $\mu(\Lambda)$, Coleman-Glashow sum rule for the low lying spin $\frac{1}{2}^{+}$baryons and $\mu\left(\Delta^{+}\right), \mu\left(\Omega^{-}\right)$for the low lying spin $\frac{3}{2}^{+}$baryons, we are able to achieve an excellent agreement with data. For the spin $\frac{1}{2}^{+}$ and spin $\frac{3}{2}^{+}$charmed baryon magnetic moments, our results are consistent with the predictions of the QCD sum rules, Light Cone sum rules and Spectral sum rules. For the cases where "light" quarks dominate in the valence structure, the sea and orbital contributions are found to be fairly significant however, they cancel in the right direction to give the correct magnitude of the total magnetic moment. On the other hand, when there is an excess of "heavy" quarks, the contribution of the "quark sea" is almost negligible, for example, $\mu\left(\Omega_{c}^{0}\right), \mu\left(\Lambda_{c}^{+}\right), \mu\left(\Xi_{c}^{+}\right), \mu\left(\Xi_{c}^{0}\right), \mu\left(\Omega_{c c}^{+}\right), \mu\left(\Omega^{-}\right)$, $\mu\left(\Omega_{c}^{* 0}\right), \mu\left(\Omega_{c c}^{*+}\right)$ and $\mu\left(\Omega_{c c c}^{*++}\right)$. The effects of configuration mixing and quark masses have also been investigated.

PACS numbers: 13.40.Em, 12.39.Fe, 14.20.Lq
\end{abstract}

\section{INTRODUCTION}

The possible size of intrinsic charm (IC) content of the nucleon [1] has been estimated to understand the phenomenological implications of the presence of heavy quarks in the nucleon. The heavy flavor charmed baryons play an important role to understand the dynamics of light quarks in the bound state as well as to understand QCD at the hadronic scale [2]. On the other hand, the static and electromagnetic properties like masses, magnetic moments etc. give valuable information regarding the internal structure of baryons [3] in the nonperturbative regime. Since there is no direct experimental data on the IC content, one has to resort to the nucleon models to obtain information on its contribution.

The magnetic moments of spin $\frac{1}{2}^{+}$, spin $\frac{3}{2}^{+}$charmed baryons and their transition magnetic moments have been considered in different approaches in literature, however, none of the phenomenological models is able to give a complete description. Calculations based on different realizations of spin-flavor symmetries have been done in the non-relativistic quark model (NRQM) [4] which have been further extended to incorporate the confinement 5], chiral symmetry with exchange currents [6] and Poincare covariance [7]. The charmed baryons magnetic moments have been investigated in the Skyrme model [8] and the bound state approach [9] considering the heavy baryons as heavy mesons bound in the field of light baryons. Recently, the charmed baryons magnetic moments have been calculated in the relativistic three-quark model where the internal quark structure of baryons is modeled by three-quark currents [10]. More recently, magnetic moments have been studied by considering the effective mass of quark bound inside the baryon [11]. The magnetic moments of spin $\frac{1}{2}^{+}$and spin $\frac{3}{2}^{+}$including transition magnetic moments of charmed baryons have been also investigated in QCD sum rules (QCDSR) [12], QCD Spectral sum rules (QSSR) [13] and light cone QCD sum rules (LCQSR) 14 16].

It would be important to mention here that the intrinsic heavy quarks are created from the quantum fluctuations associated with the bound state hadron dynamics and the process is completely determined by nonperturbative mechanisms [17]. Recently, it has been shown that one of the important model which finds application in the nonperturbative regime of QCD is the chiral constituent quark model $(\chi \mathrm{CQM})$ 18 20]. The $\chi \mathrm{CQM}$ with spin-spin generated configuration mixing 21, 22] is able to give the satisfactory explanation for the spin and flavor distribution functions 23, 24], strangeness content of the nucleon 25], weak vector and axial-vector form factors [26] etc.. When coupled with the "quark sea" polarization, orbit angular momentum of the "quark sea" (referred as Cheng and Li Mechanism) and confinement effects it is able to give a excellent fit to the octet and decuplet baryon magnetic moments and a perfect fit to the violation of Coleman-Glashow sum rule 27-30]. The quantum fluctuations generated by broken chiral symmetry in $\chi \mathrm{CQM}$ should be able to provide a viable estimate of the heavier quark flavor, for example, $c \bar{c}, b \bar{b}$ 
and $t \bar{t}$. However, it is known that these flavor fluctuations are much suppressed in the case of $b \bar{b}$ and $t \bar{t}$ as compared to the $c \bar{c}$ because the intrinsic heavy quark contributions scale as $1 / M_{q}^{2}$, where $M_{q}$ is the mass of the heavy quark 17, 31]. In this context, the scope of model was extended to the broken SU(4) symmetry which successfully predicted the important role played by IC content in determining the spin and flavor structure of the nucleon 32, 33. In the light of above investigations, it becomes desirable to broaden the scope of Ref. [33] by extending the calculations to magnetic moments and transition magnetic moments of the charmed baryons.

The purpose of the present paper is to formulate in detail the magnetic moments of spin $\frac{1}{2}+$ and spin $\frac{3}{2}+$ charmed baryons in the SU(4) framework of $\chi$ CQM. The magnetic moments of the low lying $\operatorname{spin} \frac{3}{2}+\frac{1}{2}^{+}$and $\frac{1}{2}+\rightarrow \frac{1}{2}+$ transitions as well as the transitions involving charmed baryons would also be calculated. The generalized Cheng-Li mechanism has been incorporated to calculate explicitly the contribution coming from the valence spin polarization, "quark sea" polarization and its orbital angular momentum. In order to understand the implications of charm quarks in the baryons without any valence charm quarks and to make our analysis more responsive, we would also like to calculate the low lying octet and decuplet baryons magnetic moments in the SU(4) framework of $\chi \mathrm{CQM}$. Further, it would also be interesting to examine the effects of the configuration mixing, symmetry breaking parameters, confinement effects, quark masses etc. on the magnetic moments.

The plan of work is as follows. To facilitate discussion, in Sec. 2, SU(4) $\chi$ CQM is revisited with an emphasis on the details of spin dynamics. In Sec. 3, we first present the essential details of Cheng-Li mechanism to obtain the magnetic moments of baryons and in the subsequent subsections, we discuss the baryon magnetic moments with spin $\frac{1}{2}^{+}$, spin $\frac{3}{2}^{+}$and their transition magnetic moments, respectively. Few typical cases pertaining to charmed baryons have been worked out in detail in each case. Discussion on the various inputs used in the analysis is presented in Sec. 4. In Sec. 5, we present our numerical results and their comparison with the other model predictions. Finally, we summarize our results in Sec. 6. The details pertaining to the wave functions for charmed baryons have been presented in the Appendix A.

\section{SPIN STRUCTURE IN CHIRAL CONSTITUENT QUARK MODEL}

The basic process in the $\chi \mathrm{CQM}[18]$ is the internal emission of a Goldstone Boson (GB) by a constituent quark which further splits into a $q \bar{q}$ pair as $q_{ \pm} \rightarrow \mathrm{GB}^{0}+q_{\mp}^{\prime} \rightarrow\left(q \bar{q}^{\prime}\right)+q_{\mp}^{\prime}$, where $q \bar{q}^{\prime}+q^{\prime}$ constitutes the "quark sea" [20, 23, 24, 29]. The details of $\chi \mathrm{CQM}$ in the SU(4) framework have already been discussed in literature [32, 33], however, for the sake of readability of manuscript, we discuss here the essentials of the spin structure of the baryons used in the calculations of magnetic moments.

The effective Lagrangian describing interaction between quarks and sixteen GBs, consisting of 15-plet and a singlet, can be expressed as $\mathcal{L}=g_{15} \overline{\mathbf{q}}(\Phi) \mathbf{q}$, where $g_{15}$ is the coupling constant and GBs field $\Phi$ is

$$
\Phi=\left(\begin{array}{cccc}
\frac{\pi^{0}}{\sqrt{2}}+\beta \frac{\eta}{\sqrt{6}}+\zeta \frac{\eta^{\prime}}{4 \sqrt{3}}-\gamma \frac{\eta_{c}}{4} & \pi^{+} & \alpha K^{+} & \gamma \bar{D}^{0} \\
\pi^{-} & -\frac{\pi^{0}}{\sqrt{2}}+\beta \frac{\eta}{\sqrt{6}}+\zeta \frac{\eta^{\prime}}{4 \sqrt{3}}-\gamma \frac{\eta_{c}}{4} & \alpha K^{0} & \gamma D^{-} \\
\alpha K^{-} & \alpha \bar{K}^{0} & -\beta \frac{2 \eta}{\sqrt{6}}+\zeta \frac{\eta^{\prime}}{4 \sqrt{3}}-\gamma \frac{\eta_{c}}{4} & \gamma D_{s}^{-} \\
\gamma D^{0} & \gamma D^{+} & \gamma D_{s}^{+} & -\zeta \frac{3 \eta^{\prime}}{4 \sqrt{3}}+\gamma \frac{3 \eta_{c}}{4}
\end{array}\right) .
$$

$\mathrm{SU}(4)$ symmetry breaking is introduced by considering $M_{c}>M_{s}>M_{u, d}$ as well as by considering the masses of GBs to be nondegenerate $\left(M_{\eta_{c}}>M_{\eta^{\prime}}>M_{K, \eta}>M_{\pi}\right)$ [20, 32, 33]. The parameter $a\left(=\left|g_{15}\right|^{2}\right)$ denotes the transition probability of chiral fluctuation of the splitting $u(d) \rightarrow d(u)+\pi^{+(-)}$, whereas $a \alpha^{2}, a \beta^{2}$, $a \zeta^{2}$ and $a \gamma^{2}$ denote the probabilities of transitions of $u(d) \rightarrow s+K^{-(o)}, u(d, s) \rightarrow u(d, s)+\eta, u(d, s) \rightarrow u(d, s)+\eta^{\prime}$ and $u(d) \rightarrow c+\bar{D}^{0}\left(D^{-}\right)$, respectively.

The spin structure of the baryon is defined as [20, 23, 29]

$$
\widehat{B} \equiv\langle B|\mathcal{N}| B\rangle,
$$

where $|B\rangle$ is the baryon wave function and $\mathcal{N}$ is the number operator defined as

$$
\mathcal{N}=n_{u_{+}} u_{+}+n_{u_{-}} u_{-}+n_{d_{+}} d_{+}+n_{d_{-}} d_{-}+n_{s_{+}} s_{+}+n_{s_{-}} s_{-}+n_{c_{+}} c_{+}+n_{c_{-}} c_{-}
$$

$n_{q_{ \pm}}$being the number of $q_{ \pm}$quarks. The valence spin polarizations $\left(\Delta q_{\mathrm{val}}=q_{+}-q_{-}\right)$for a given baryon can be calculated using the spin and flavor wave functions detailed in Appendix A. The "quark sea" spin polarizations $\left(\Delta q_{\text {sea }}\right)$ can be calculated by substituting for each valence quark

$$
q_{ \pm} \rightarrow \sum P_{q} q_{ \pm}+\left|\psi\left(q_{ \pm}\right)\right|^{2}
$$


where $\sum P_{q}$ is the probability of emission of GBs from a $q$ quark and $\left|\psi\left(q_{ \pm}\right)\right|^{2}$ is the probability of transforming a $q_{ \pm}$ quark [33].

\section{MAGNETIC MOMENTS IN $\chi$ CQM}

The magnetic moment of a given baryon receives contributions from the valence quarks, "quark sea" and orbital angular momentum of the "quark sea" following Cheng and Li [20, 25, 28, 30] and is expressed as

$$
\mu(B)_{\text {total }}=\mu(B)_{\mathrm{val}}+\mu(B)_{\mathrm{sea}}+\mu(B)_{\text {orbit }},
$$

where $\mu(B)_{\text {val }}$ and $\mu(B)_{\text {sea }}$ represent the contributions of the valence quarks and the "quark sea" to the magnetic moments due to spin polarizations. The term $\mu(B)_{\text {orbit }}$ corresponds to the orbital angular momentum contribution of the "quark sea".

In terms of quark magnetic moments and spin polarizations, the valence, sea and orbital contributions can be defined as

$$
\mu(B)_{\mathrm{val}}=\sum_{q=u, d, s, c} \Delta q_{\mathrm{val}} \mu_{q}, \quad \mu(B)_{\mathrm{sea}}=\sum_{q=u, d, s, c} \Delta q_{\mathrm{sea}} \mu_{q}, \mu(B)_{\mathrm{orbit}}=\sum_{q=u, d, s, c} \Delta q_{\mathrm{val}} \mu\left(q_{+} \rightarrow q_{-}^{\prime}\right),
$$

where $\mu_{q}=\frac{e_{q}}{2 M_{q}}(q=u, d, s, c)$ is the quark magnetic moment, $\mu\left(q_{+} \rightarrow q_{-}^{\prime}\right)$ is the orbital moment for any chiral fluctuation, $e_{q}$ and $M_{q}$ are the electric charge and the mass respectively for the quark $q$.

The valence and sea quark spin polarizations $\left(\Delta q_{\text {val }}\right.$ and $\left.\Delta q_{\text {sea }}\right)$ for a given baryon can be calculated as discussed in the previous section and Ref. [30]. The orbital angular momentum contribution of each chiral fluctuation is given as $[28,[30]$

$$
\mu\left(q_{+} \rightarrow q_{-}^{\prime}\right)=\frac{e_{q^{\prime}}}{2 M_{q}}\left\langle l_{q}\right\rangle+\frac{e_{q}-e_{q^{\prime}}}{2 M_{\mathrm{GB}}}\left\langle l_{\mathrm{GB}}\right\rangle,
$$

where $\left\langle l_{q}\right\rangle=\frac{M_{\mathrm{GB}}}{M_{q}+M_{\mathrm{GB}}}$ and $\left\langle l_{\mathrm{GB}}\right\rangle=\frac{M_{q}}{M_{q}+M_{\mathrm{GB}}}$. The quantities $\left(l_{q}, l_{\mathrm{GB}}\right)$ and $\left(M_{q}, M_{\mathrm{GB}}\right)$ are the orbital angular momenta and masses of quark and GBs, respectively. The orbital moment of each process in Eq.(7) is then multiplied by the probability for such a process to take place to yield the magnetic moment due to all the transitions starting with a given valence quark. The details of the orbital moment in the $\mathrm{SU}(3)$ framework has already been worked out in Ref. [30]. In this work, we extend our calculations to include the contribution from $c \bar{c}$ fluctuations. For example,

$$
\begin{gathered}
{\left[\mu\left(u_{ \pm} \rightarrow\right)\right]= \pm a\left[\left(\frac{1}{2}+\frac{\beta^{2}}{6}+\frac{\zeta^{2}}{48}+\frac{\gamma^{2}}{16}\right) \mu\left(u_{+} \rightarrow u_{-}\right)+\mu\left(u_{+} \rightarrow d_{-}\right)+\alpha^{2} \mu\left(u_{+} \rightarrow s_{-}\right)+\gamma^{2} \mu\left(u_{+} \rightarrow c_{-}\right)\right]} \\
{\left[\mu\left(d_{ \pm} \rightarrow\right)\right]= \pm a\left[\mu\left(d_{+} \rightarrow u_{-}\right)+\left(\frac{1}{2}+\frac{\beta^{2}}{6}+\frac{\zeta^{2}}{48}+\frac{\gamma^{2}}{16}\right) \mu\left(d_{+} \rightarrow d_{-}\right)+\alpha^{2} \mu\left(d_{+} \rightarrow s_{-}\right)+\gamma^{2} \mu\left(d_{+} \rightarrow c_{-}\right)\right],} \\
{\left[\mu\left(s_{ \pm} \rightarrow\right)\right]= \pm a\left[\alpha^{2} \mu\left(s_{+} \rightarrow u_{-}\right)+\alpha^{2} \mu\left(s_{+} \rightarrow d_{-}\right)+\left(\frac{2}{3} \beta^{2}+\frac{\zeta^{2}}{48}+\frac{\gamma^{2}}{16}\right) \mu\left(s_{+} \rightarrow s_{-}\right)+\gamma^{2} \mu\left(s_{+} \rightarrow c_{-}\right)\right],}
\end{gathered}
$$

and

$$
\left[\mu\left(c_{ \pm} \rightarrow\right)\right]= \pm a\left[\gamma^{2} \mu\left(c_{+} \rightarrow u_{-}\right)+\gamma^{2} \mu\left(c_{+} \rightarrow d_{-}\right)+\gamma^{2} \mu\left(c_{+} \rightarrow s_{-}\right)+\left(\frac{3}{16} \zeta^{2}+\frac{9}{16} \gamma^{2}\right) \mu\left(c_{+} \rightarrow c_{-}\right)\right]
$$

The above equations can easily be generalized by including the coupling breaking and mass breaking terms. The orbital moments of $u, d, s$ and $c$ quarks in terms of the $\chi \mathrm{CQM}$ parameters $(a, \alpha, \beta, \zeta, \gamma)$, quark masses $\left(M_{u}, M_{d}, M_{s}, M_{c}\right)$ and GB masses $\left(M_{\pi}, M_{k}, M_{\eta}, M_{\eta^{\prime}}, M_{D}, M_{D_{s}}, M_{\eta_{c}}\right)$, are respectively given as

$$
\begin{aligned}
{\left[\mu\left(u_{+} \rightarrow\right)\right]=} & a\left[\frac{3 M_{u}^{2}}{2 M_{\pi}\left(M_{u}+M_{\pi}\right)}-\frac{\alpha^{2}\left(M_{K}^{2}-3 M_{u}^{2}\right)}{2 M_{K}\left(M_{u}+M_{K}\right)}+\frac{\gamma^{2} M_{D}}{\left(M_{u}+M_{D}\right)}\right. \\
& \left.+\frac{\beta^{2} M_{\eta}}{6\left(M_{u}+M_{\eta}\right)}+\frac{\zeta^{2} M_{\eta^{\prime}}}{48\left(M_{u}+M_{\eta^{\prime}}\right)}+\frac{\gamma^{2} M_{\eta_{c}}}{16\left(M_{u}+M_{\eta_{c}}\right)}\right] \mu_{N}, \\
{\left[\mu\left(d_{+} \rightarrow\right)\right]=} & a \frac{M_{u}}{M_{d}}\left[\frac{3\left(M_{\pi}^{2}-2 M_{d}^{2}\right)}{4 M_{\pi}\left(M_{d}+M_{\pi}\right)}-\frac{\alpha^{2} M_{K}}{2\left(M_{d}+M_{K}\right)}+\frac{\gamma^{2}\left(2 M_{D}^{2}-3 M_{d}^{2}\right)}{2 M_{D}\left(M_{d}+M_{D}\right)}\right. \\
& \left.-\frac{\beta^{2} M_{\eta}}{12\left(M_{d}+M_{\eta}\right)}-\frac{\zeta^{2} M_{\eta^{\prime}}}{96\left(M_{d}+M_{\eta^{\prime}}\right)}-\frac{\gamma^{2} M_{\eta_{c}}}{32\left(M_{d}+M_{\eta_{c}}\right)}\right] \mu_{N},
\end{aligned}
$$




$$
\begin{gathered}
{\left[\mu\left(s_{+} \rightarrow\right)\right]=a \frac{M_{u}}{M_{s}}\left[\frac{\alpha^{2}\left(M_{K}^{2}-3 M_{s}^{2}\right)}{2 M_{K}\left(M_{s}+M_{K}\right)}+\frac{\gamma^{2}\left(2 M_{D_{s}}^{2}-3 M_{s}^{2}\right)}{2 M_{D_{s}}\left(M_{s}+M_{D_{s}}^{2}\right)}-\frac{\beta^{2} M_{\eta}}{3\left(M_{s}+M_{\eta}\right)}-\frac{\zeta^{2} M_{\eta^{\prime}}}{96\left(M_{s}+M_{\eta^{\prime}}\right)}-\frac{\gamma^{2} M_{\eta_{c}}}{32\left(M_{s}+M_{\eta_{c}}\right)}\right] \mu_{N},} \\
{\left[\mu\left(c_{+} \rightarrow\right)\right]=a \frac{M_{u}}{M_{c}}\left[\frac{\gamma^{2}\left(M_{D}^{2}+3 M_{c}^{2}\right)}{2 M_{D}\left(M_{c}+M_{D}^{2}\right)}-\frac{\gamma^{2}\left(M_{D_{s}}^{2}-3 M_{c}^{2}\right)}{2 M_{D_{s}}\left(M_{c}+M_{D_{s}}^{2}\right)}+\frac{3 \zeta^{2} M_{\eta^{\prime}}}{16\left(M_{c}+M_{\eta^{\prime}}\right)}+\frac{9 \gamma^{2} M_{\eta_{c}}}{16\left(M_{c}+M_{\eta_{c}}\right)}\right] \mu_{N},}
\end{gathered}
$$

where $\mu_{N}$ is the nuclear magneton.

After discussing the general formalism to calculate the valence, sea and orbital contributions to the magnetic moments, we now discuss the explicit calculations for the low lying and charmed spin $\frac{1}{2}+$ and spin $\frac{3}{2}+$ baryons as well as their transition magnetic moments.

\subsection{Magnetic moments of spin $\frac{1}{2}^{+}$baryons}

The magnetic moments of all the spin $\frac{1}{2}^{+}$baryons can be calculated using Eq.(5). The spin structure of a spin $\frac{1}{2}^{+}$ baryon (from Appendix A) is given as

$$
\hat{B} \equiv\langle B|\mathcal{N}| B\rangle=\cos ^{2} \phi\left\langle 120,{ }^{2} 20_{M}|\mathcal{N}| 120,{ }^{2} 20_{M}\right\rangle_{B}+\sin ^{2} \phi\left\langle 168,{ }^{2} 20_{M}|\mathcal{N}| 168,{ }^{2} 20_{M}\right\rangle_{B} .
$$

In this section, as an example, we detail the calculations of the one single and one double charmed baryon $\Xi_{c}^{\prime}+$ and $\Xi_{c c}^{++}$.

The valence spin structure for the single charmed baryon $\Xi_{c}^{\prime}+$ can be expressed as

$$
\widehat{\Xi}_{c}^{\prime}=\cos ^{2} \phi\left(\frac{5}{6} u_{+}+\frac{1}{6} u_{-}+\frac{5}{6} s_{+}+\frac{1}{6} s_{-}+\frac{1}{3} c_{+}+\frac{2}{3} c_{-}\right)+\sin ^{2} \phi\left(\frac{2}{3} u_{+}+\frac{1}{3} u_{-}+\frac{2}{3} s_{+}+\frac{1}{3} s_{-}+\frac{2}{3} c_{+}+\frac{1}{3} c_{-}\right),
$$

leading to the valence contribution to the magnetic moment of $\Xi_{c}^{\prime+}$

$$
\mu\left(\Xi_{c}^{\prime}+\right)_{\text {val }}=\cos ^{2} \phi\left(\frac{2}{3} \mu_{u}+\frac{2}{3} \mu_{s}-\frac{1}{3} \mu_{c}\right)+\sin ^{2} \phi\left(\frac{1}{3} \mu_{u}+\frac{1}{3} \mu_{s}+\frac{1}{3} \mu_{c}\right) .
$$

The spin structure of the "quark sea" $\left(\Delta q_{\text {sea }}\right)$ can be calculated by substituting Eq.(44) for every valence quark in Eq.(17), leading to the "quark sea" contribution to the magnetic moment of $\Xi_{c}^{\prime+}$ expressed as

$$
\begin{aligned}
\mu\left(\Xi_{c}^{\prime}\right)_{\text {sea }}= & -\frac{a}{3} \cos ^{2} \phi\left[\left(4+4 \alpha^{2}+\frac{2}{3} \beta^{2}+\frac{\zeta^{2}}{12}+\frac{9}{8} \gamma^{2}\right) \mu_{u}+\left(2+2 \alpha^{2}-\gamma^{2}\right) \mu_{d}+\left(6 \alpha^{2}+\frac{8}{3} \beta^{2}+\frac{\zeta^{2}}{12}+\frac{9}{8} \gamma^{2}\right) \mu_{s}\right. \\
& \left.-\frac{1}{8}\left(3 \zeta^{2}+\gamma^{2}\right) \mu_{c}\right]-\frac{a}{3} \sin ^{2} \phi\left[\left(2+2 \alpha^{2}+\frac{\beta^{2}}{3}+\frac{\zeta^{2}}{24}+\frac{33}{16} \gamma^{2}\right) \mu_{u}+\left(1+\alpha^{2}+\gamma^{2}\right) \mu_{d}\right. \\
& \left.+\left(3 \alpha^{2}+\frac{4}{3} \beta^{2}+\frac{\zeta^{2}}{24}+\frac{33}{16} \zeta^{2}\right) \mu_{s}+\frac{1}{8}\left(3 \zeta^{2}+49 \gamma^{2}\right) \mu_{c}\right] .
\end{aligned}
$$

The orbital contribution of the "quark sea" to the total magnetic moment of $\Xi_{c}^{\prime}$, obtained using Eqs.(66) and (18), can be expressed as

$$
\mu\left(\Xi_{c}^{\prime}\right)_{\text {orbit }}=\cos ^{2} \phi\left(\frac{2}{3} \mu\left(u_{+} \rightarrow\right)+\frac{2}{3} \mu\left(s_{+} \rightarrow\right)-\frac{1}{3} \mu\left(c_{+} \rightarrow\right)\right)+\sin ^{2} \phi\left(\frac{1}{3} \mu\left(u_{+} \rightarrow\right)+\frac{1}{3} \mu\left(s_{+} \rightarrow\right)+\frac{1}{3} \mu\left(c_{+} \rightarrow\right)\right) .
$$

Substituting the valence, sea and orbital contribution from Eqs.(18), (19) and (20) in Eq.(15), we can calculate the total magnetic moment of $\Xi_{c}^{\prime+}$.

For the double charmed baryon $\Xi_{c c}^{++}$, the valence spin structure can be expressed as

$$
\widehat{\Xi}_{c c}^{++}=\cos ^{2} \phi\left(\frac{1}{3} u_{+}+\frac{2}{3} u_{-}+\frac{5}{3} c_{+}+\frac{1}{3} c_{-}\right)+\sin ^{2} \phi\left(\frac{2}{3} u_{+}+\frac{1}{3} u_{-}+\frac{4}{3} c_{+}+\frac{2}{3} c_{-}\right),
$$


giving the valence, sea and orbital contribution to the magnetic moment of $\Xi_{c c}^{++}$as

$$
\begin{aligned}
\mu\left(\Xi_{c c}^{++}\right)_{\mathrm{val}} & =\cos ^{2} \phi\left(-\frac{1}{3} \mu_{u}+\frac{4}{3} \mu_{c}\right)+\sin ^{2} \phi\left(\frac{1}{3} \mu_{u}+\frac{2}{3} \mu_{c}\right) \\
\mu\left(\Xi_{c c}^{++}\right)_{\mathrm{sea}} & =\frac{a}{3} \cos ^{2} \phi\left[\left(2+\alpha^{2}+\frac{\beta^{2}}{3}+\frac{\zeta^{2}}{24}-\frac{47}{16} \gamma^{2}\right) \mu_{u}+\left(1-4 \gamma^{2}\right) \mu_{d}+\left(\alpha^{2}-4 \gamma^{2}\right) \mu_{s}-\frac{1}{2}\left(3 \zeta^{2}+31 \gamma^{2}\right) \mu_{c}\right] \\
& -\frac{a}{3} \sin ^{2} \phi\left[\left(2+\alpha^{2}+\frac{\beta^{2}}{3}+\frac{\zeta^{2}}{24}+\frac{49}{16} \gamma^{2}\right) \mu_{u}+\left(1+2 \gamma^{2}\right) \mu_{d}+\left(\alpha^{2}+2 \gamma^{2}\right) \mu_{s}+\frac{1}{4}\left(3 \zeta^{2}+37 \gamma^{2}\right) \mu_{c}\right] \\
\mu\left(\Xi_{c c}^{++}\right)_{\text {orbit }} & =\cos ^{2} \phi\left(-\frac{1}{3} \mu\left(u_{+} \rightarrow\right)+\frac{4}{3} \mu\left(c_{+} \rightarrow\right)\right)+\sin ^{2} \phi\left(\frac{1}{3} \mu\left(u_{+} \rightarrow\right)+\frac{2}{3} \mu\left(c_{+} \rightarrow\right)\right) .
\end{aligned}
$$

The valence, sea and orbital contribution from Eqs.(23), (24) and (24) give the total magnetic moment of $\Xi_{c c}^{++}$. Similarly, one can calculate the valence, sea and orbital contributions to the magnetic moments of all the spin $\frac{1}{2}^{+}$ baryons. The expressions for the valence and sea contributions to the magnetic moments of the low lying and charmed spin $\frac{1}{2}^{+}$baryons have been presented in the Table \

\subsection{Magnetic moments of spin $\frac{3}{2}^{+}$baryons}

In this section, we detail the calculations of magnetic moments of spin $\frac{3}{2}+$ baryons by taking the example of a charmed baryon $\Xi_{c}^{*+}$. From Appendix A, the spin structure of a spin $\frac{3}{2}+$ baryon is given as

$$
\widehat{B}^{*} \equiv\left\langle B^{*}|\mathcal{N}| B^{*}\right\rangle=\left\langle 120,{ }^{4} 20_{S}|\mathcal{N}| 120,{ }^{4} 20_{S}\right\rangle_{B^{*}} .
$$

The valence spin structure of $\Xi_{c}^{*+}$ can be expressed as

$$
\widehat{\Xi}_{c}^{*+}=u_{+}+s_{+}+c_{+},
$$

giving the valence contribution to the magnetic moment as

$$
\mu\left(\Xi_{c}^{*+}\right)_{\mathrm{val}}=\mu_{u}+\mu_{s}+\mu_{c} .
$$

The "quark sea" contribution to the magnetic moment of $\Xi_{c}^{* 0}$ can be calculated by substituting Eq.(4) for every valence quark in Eq.(26), giving the sea contribution as

$$
\begin{aligned}
\mu\left(\Xi_{c}^{*+}\right)_{\text {sea }} & =-a\left[\left(2+2 \alpha^{2}+\frac{\beta^{2}}{3}+\frac{\zeta^{2}}{24}+\frac{33}{16} \gamma^{2}\right) \mu_{u}+\left(1+\alpha^{2}+\gamma^{2}\right) \mu_{d}\right. \\
& \left.+\left(3 \alpha^{2}+\frac{4}{3} \beta^{2}+\frac{\zeta^{2}}{24}+\frac{33}{16} \gamma^{2}\right) \mu_{s}+\frac{1}{8}\left(3 \zeta^{2}+49 \gamma^{2}\right) \mu_{c}\right]
\end{aligned}
$$

The orbital angular momentum contribution to the magnetic moment of $\Xi_{c}^{*+}$ is given as

$$
\mu\left(\Xi_{c}^{*+}\right)_{\text {orbit }}=\mu\left(u_{+} \rightarrow\right)+\mu\left(s_{+} \rightarrow\right)+\mu\left(c_{+} \rightarrow\right) .
$$

Substituting the valence, sea and orbital contribution from Eqs.(27), (28) and (29) in Eq.(15), we can calculate the total magnetic moment of $\Xi_{c}^{*+}$. The valence, sea and orbital contribution to the magnetic moments of other spin $\frac{3}{2}^{+}$ charmed baryons can similarly be calculated and the expressions for the valence and sea contribution to the total magnetic moment of the spin $\frac{3}{2}^{+}$charmed baryons have been presented in Table

\subsection{Transition magnetic moments}

In this section, we calculate the transition magnetic moments for the radiative decays $B_{i} \rightarrow B_{f}+\gamma$, where $B_{i}$ and $B_{f}$ are the initial and final baryons, for the spin $\frac{3}{2}^{+} \rightarrow \frac{1}{2}^{+}$and $\frac{1}{2}^{+} \rightarrow \frac{1}{2}^{+}$transitions of the baryons. In particular, the transition magnetic moments considered in this work are for spin $\frac{3}{2}+\rightarrow \frac{1}{2}+$ transitions corresponding to the charmless decuplet to octet transitions $(10 \rightarrow 8)$, single charmed sextet to anti-triplet transitions $(6 \rightarrow \overline{3})$, single charmed sextet to sextet transitions $(6 \rightarrow 6)$ and double charmed triplet to triplet transitions $(3 \rightarrow 3)$ transitions. On the other hand, 
the spin $\frac{1}{2}^{+} \rightarrow \frac{1}{2}^{+}$transitions considered are for the charmless octet to octet transitions $(8 \rightarrow 8)$ and single charmed anti-triplet to sextet transitions $(\overline{3} \rightarrow 6)$. The details of the structure have been presented in Appendix A.

The transition magnetic moment can be calculated from the matrix element

$$
\widehat{B_{i} B_{f}}(\mathbf{k})=\left\langle B_{f}, J_{z}=\frac{1}{2}\left|\mathcal{N} e^{-\iota k . z}\right| B_{i}, J_{z}=\frac{1}{2}\right\rangle
$$

where $\mathbf{k}$ is the momentum of the photon. As an example, we discuss here the case of transition magnetic moment of the $6 \rightarrow 6$ transition $\left(\Xi_{c}^{*+} \Xi_{c}^{\prime+}\right)$. The spin structure for the $\left(\Xi_{c}^{*+} \Xi_{c}^{\prime}\right)$ transition is given as

$$
\widehat{\Xi_{c}^{*+} \Xi_{c}^{\prime}}+(\mathbf{k})=\frac{\sqrt{2}}{3}\left(-u_{+}-s_{+}+2 c_{+}\right) \cdot e^{-\frac{1}{6} k^{2} R^{2}}
$$

giving the valence contribution to the magnetic moment of $\left(\Xi_{c}^{*+} \Xi_{c}^{\prime}\right)$ transition as

$$
\mu\left(\Xi_{c}^{*+} \Xi_{c}^{\prime}\right)_{\mathrm{val}}=\frac{\sqrt{2}}{3}\left(-\mu_{u}-\mu_{s}+2 \mu_{c}\right) \cdot e^{-\frac{1}{6} k^{2} R^{2}} .
$$

The "quark sea" contribution can be calculated by making substitution Eq.(4) for every valence quark. The quark sea contribution for the magnetic moment of $\left(\Xi_{c}^{*+} \Xi_{c}^{\prime+}\right)$ transition is then expressed as

$$
\begin{aligned}
\mu\left(\Xi_{c}^{*+} \Xi_{c}^{\prime}\right)_{\text {sea }} & =\frac{\sqrt{2}}{3} a\left[\left(2+2 \alpha^{2}+\frac{\beta^{2}}{3}+\frac{\zeta^{2}}{24}-\frac{15}{16} \gamma^{2}\right) \mu_{u}+\left(1+\alpha^{2}-2 \gamma^{2}\right) \mu_{d}\right. \\
& \left.+\left(3 \alpha^{2}+\frac{4}{3} \beta^{2}+\frac{\zeta^{2}}{24}-\frac{15}{16} \gamma^{2}\right) \mu_{s}-\frac{1}{4}\left(3 \zeta^{2}+25 \gamma^{2}\right) \mu_{c}\right] \cdot e^{-\frac{1}{6} k^{2} R^{2}} .
\end{aligned}
$$

The orbital angular momentum contribution in this case is

$$
\mu\left(\Xi_{c}^{*+} \Xi_{c}^{\prime+}\right)_{\text {orbit }}=\frac{\sqrt{2}}{3}\left(-\mu\left(u_{+} \rightarrow\right)-\mu\left(s_{+} \rightarrow+2 \mu\left(c_{+} \rightarrow\right)\right) \cdot e^{-\frac{1}{6} k^{2} R^{2}} .\right.
$$

The total magnetic moment for the transition $\left(\Xi_{c}^{*+} \Xi_{c}^{\prime}\right)$ can be calculated by adding Eqs.(32), (333) and (34). The detailed expressions for the valence, sea and orbital contribution to the magnetic moments for all other transitions can be calculated similarly and the expressions are presented in the Table III.

\section{INPUT PARAMETERS}

In this section, we discuss the various input parameters needed for the numeric calculation of the magnetic moments of spin $\frac{1}{2}^{+}$and spin $\frac{3}{2}^{+}$baryons. The valence, sea and orbital contributions to the magnetic moment in $\chi \mathrm{CQM}$ with $\mathrm{SU}(4)$ broken symmetry involve the symmetry breaking parameters and mixing angle $\phi$. The symmetry breaking parameters $a, a \alpha^{2}, a \beta^{2}, a \zeta^{2}, a \gamma^{2}$ representing respectively, the probabilities of fluctuations of a constituent quark into pions, $K, \eta, \eta^{\prime}, \eta_{c}$, are expected to follow the hierarchy $a>a \alpha^{2}>a \beta^{2}>a \zeta^{2}>a \gamma^{2}$ as they are dominated by the mass differences. As a consequence, the probability of emitting a heavier meson such as $D$ from a lighter quark is much smaller than that of emitting the light meson such as $K, \eta$ and $\eta^{\prime}$ etc.. The symmetry breaking parameters are usually fixed by the spin polarization functions $\Delta u, \Delta d$ and $Q^{2}$ independent parameter $\Delta_{3}(=\Delta u-\Delta d)[34-36]$ as well as the flavor distribution functions $\bar{u}-\bar{d}$ and $\bar{u} / \bar{d}$ 37, 38], measured from the deep inelastic scattering experiments. The mixing angle $\phi$ is fixed by fitting neutron charge radius [39]. A fine grained analysis with the symmetry breaking lead to the following set of symmetry breaking parameters as the best fit

$$
a=0.12, \quad \alpha \simeq \beta=0.45, \quad \zeta=-0.21, \text { and } \gamma=0.11
$$

In addition to the parameters of $\chi \mathrm{CQM}$ and mixing angle $\phi$ as discussed above, the orbital angular momentum contributions are characterized by the quark, GB masses and the harmonic-oscillator radius parameter $R$. For evaluating their contribution, we have used their on shell mass values in accordance with several other similar calculations [22, 40]. For the constituent quark masses $u, d, s, c$, we have used their widely accepted values in hadron spectroscopy $M_{u}=M_{d}=0.33 \mathrm{GeV}, M_{s}=0.51 \mathrm{GeV}, M_{c}=1.70 \mathrm{GeV}$. The quark masses and corresponding magnetic moments have to be further adjusted by the quark confinement effects [30, 41]. For the low lying baryons, Kerbikov et al. [42] have given a successful description of the magnetic moment with confinement effects playing a leading role. However, in the present case the simplest way to incorporate this adjustment [41] is to first express $M_{q}$ in the magnetic moment operator in terms of $M_{B}$, the mass of the baryon obtained additively from the quark masses, which then is replaced by $M_{B}+\Delta M, \Delta M$ being the mass difference between the experimental value and $M_{B}$. This leads to the following adjustments in the quark magnetic moments: $\mu_{u}=2\left[1-\left(\Delta M / M_{B}\right)\right] \mu_{N}, \mu_{d}=-\left[1-\left(\Delta M / M_{B}\right)\right] \mu_{N}$, $\mu_{s}=-M_{u} / M_{s}\left[1-\left(\Delta M / M_{B}\right)\right] \mu_{N}$ and $\mu_{c}=2 M_{u} / M_{c}\left[1-\left(\Delta M / M_{B}\right)\right] \mu_{N}$. 


\section{RESULTS AND DISCUSSION}

The parameters discussed above have been used to calculate the various spin polarization functions, non-singlet components $\Delta_{3}$ and $\Delta_{8}$ and flavor distribution functions in $\mathrm{SU}(4) \chi \mathrm{CQM}$. The values obtained for the case of proton are as follows

$$
\begin{gathered}
\Delta u=0.93, \Delta d=-0.34, \Delta s=-0.03, \Delta c=-0.002, \Delta_{3}=1.2696, \Delta_{8}=0.64, \\
\bar{u}=0.23, \bar{d}=0.34, \bar{s}=0.086, \bar{c}=0.005, \bar{u}-\bar{d}=-0.11, \frac{\bar{d}}{\bar{u}}=1.49 .
\end{gathered}
$$

We find that a fairly good fit is achieved in the parameters listed above when compared with the latest data [3, 17, 35]. In particular, the agreement corresponding to the strangeness and intrinsic charm contribution to the nucleon in terms of the magnitude as well as the sign is quite satisfactory when compared with the latest data [3, 17, 35]. A detailed implications of these parameters have already been discussed in Ref. [33]. It is interesting to mention here that these strangeness and charm related parameters have not been taken as inputs in our calculations and still a satisfactory agreement is obtained. In addition, $\mathrm{SU}(4) \chi \mathrm{CQM}$ leads to many new predictions on observables which are directly related to the charm content of nucleon and are found to be almost an order of magnitude smaller than the strange quark contributions but not entirely insignificant. Consistency of these charm related parameters can be checked by future experiments.

The spin polarization functions discussed above have been used to calculate the baryon magnetic moments. In Tables [V and V] we have presented the results for the magnetic moments of low lying and charmed spin $\frac{1}{2}^{+}$, spin $\frac{3}{2}^{+}$baryons. In Table VI] we have presented the magnetic moments for the low lying spin $\frac{3}{2}^{+} \rightarrow \frac{1}{2}^{+}$and $\frac{1}{2}^{+} \rightarrow \frac{1}{2}^{+}$ transitions as well as the transitions involving charmed baryons. In the tables, we have presented the explicit results for the valence, sea and orbital contributions to the magnetic moments. We have also compared our results with the predictions of NRQM [4], Lattice QCD [43] and recent experimental data available [3]. Since there is no experimental information available for charmed baryon magnetic moments, we have presented the predictions of QCD sum rules (QCDSR) [12], QCD Spectral sum rules (QSSR) [13], Light Cone QCD sum rules (LCQSR) [14 16].

A cursory look at the tables reveal that the our results are smaller than the NRQM predictions in most of the cases and our results are not only in agreement with available data but also show improvement over other models in most of the cases where the experimental data is available. On the other hand, for the case of the magnetic moments where experimental data is not available, our results are consistent with the results of QCDSR, QSSR, LCQSR as well as with the other models existing in literature. One can also observe that the orbital part contributes with the same sign as valence quark distribution, while the sea part contribute with the opposite sign making the sea and orbital contributions significant. The sum of residual "quark sea" and valence quark contribution give the magnetic moment of baryons.

From Table IV, when we compare our results for the spin $\frac{1}{2}^{+}$baryons with the available experimental data as well as the other model calculations, we find that our model is able to get a fairly good account of the most of magnetic moments, wherever the experimental data is available. Presently, experimental information is available for the low lying octet baryons and violation of Coleman-Glashow sum rule $(\Delta \mathrm{CG})$ 27]. It is interesting to observe that our results for the magnetic moments of $p, \Sigma^{+}, \Xi^{0}$ and $\Lambda$ give a perfect fit to the experimental values [3] whereas for all other octet baryons our predictions are within $10 \%$ of the observed values. Besides this, we have also been able to get an excellent fit to $\Delta \mathrm{CG}$. The fit becomes all the more impressive when it is realized that none of the magnetic moments are used as inputs and $\triangle \mathrm{CG}$ can be described without resorting to additional parameters.

A closer look at the table reveals that if an attempt is made to explain the contribution of the orbital angular momentum of the "quark sea", we find the contribution of orbital angular momentum to be as important as that of the "quark sea" contribution through the spin polarization of the $q \bar{q}$ pairs. In fact, the sea and orbital contributions are fairly significant as compared to the valence contributions and they cancel in the right direction giving the right magnitude of the total magnetic moment. For example, the valence contributions of $p, \Sigma^{+}$and $\Xi^{0}$ are higher in magnitude than the experimental value but the sea contribution being higher in magnitude than the orbital contribution reduces the valence contribution leading to a better agreement with data. Similarly, in the case of $n$, $\Sigma^{-}$and $\Xi^{-}$the valence contribution in magnitude is lower than the experimental value but in these cases the sea contribution is lower than the orbital part so it adds on to the valence contribution again improving agreement with data. It is important to mention here that the IC contribution to the proton spin polarizations and hence magnetic moments is quite small so the predictions of the $\mathrm{SU}(4) \chi \mathrm{CQM}$ do not differ significantly from our earlier results in the $\mathrm{SU}(3) \chi \mathrm{CQM}$ for the octet baryons [30].

In the case of charmed baryons also, there is a significant contribution from the "quark sea" spin and orbital angular momentum. Only in the case of $\Omega_{c}^{0}, \Lambda_{c}^{+}, \Xi_{c}^{+}, \Xi_{c}^{0}$ and $\Omega_{c c}^{+}$, the magnetic moment if dominated by the valence 
contribution as the sea and orbital contributions are quite small in magnitude. This is because of the fact that the above mentioned baryons are dominated by the "heavy" quarks in the valence structure. Thus, in a very interesting manner, the orbital and sea contributions together add on to the valence contributions leading to better agreement with data. This not only endorses the earlier conclusion of Cheng and Li [28] but also suggests that the Cheng-Li mechanism could perhaps provide the dominant dynamics of the constituents in the nonperturbative regime of QCD on which further corrections could be evaluated.

From Table V], we can compare our results for the low lying as well as charmed spin $\frac{3}{2}+$ baryons with other model calculations as well as with the available experimental data. In this case also, we have presented the explicit results for the valence, sea and the orbital contributions. For the magnetic moments of the low lying decuplet baryons, only three experimental results are presently available. Our predicted value for $\Delta^{++}=4.51$, is well within the experimental range $3.7 \sim 7.5[3]$. Similarly, for the case of $\Delta^{+}$and $\Omega^{-}$our predicted values 2.0 and -1.71 , agree with the experimentally observed values $\left(2.7_{-1.3}^{+1.0} \pm 1.5 \pm 3[44]\right.$ and $-1.94 \pm 0.31$ [45], respectively). For all other baryons our predictions are consistent with the predictions of the QCDSR [12], LCQSR [15], Lattice QCD [43] and other models existing in literature. However, there is a small discrepancy in the case of $\Sigma^{* 0}$ magnetic moment while comparing our results with other model calculations. In this case, the contribution of the orbital part is negligible and the valence and sea contributions are of the same order. The valence and sea contribution being of opposite signs cancel each other completely leading to a very small $\Sigma^{* 0}$. Any experimental data on $\Sigma^{* 0}$ would have important implications for the Cheng-Li mechanism. For the charmed spin $\frac{3}{2}+$ baryons, since there is no experimental information available, we have compare our results with the predictions of the LCQSR [15]. Our results are consistent with their predictions and also with the other models existing in the literature [11, 12].

On the closer scrutiny of the results we find that in the cases where there is an excess of up and down quarks in the valence structure, the contribution of the "quark sea" and its orbital angular momentum is quite significant when compared with the valence contribution. On the other hand, when there is an excess of strange and charm quarks in the valence structure, the contribution of the "quark sea" and its orbital angular momentum is almost negligible as compared to the valence contribution. This can be easily understood when we compare the sea and orbital contributions of $\Omega^{-}, \Omega_{c}^{* 0}, \Omega_{c c}^{*+}$ and $\Omega_{c c c}^{*++}$ with the sea and orbital contributions of the other baryons. In these cases, the total magnetic moment is more or less the same as the valence contribution whereas in all other cases there is a significant contribution from the resultant sea and orbital contributions. It would be interesting to mention here that this is due to the fact that the strange and charm contribution to the magnetic moment is almost an order of magnitude smaller than the up and down quarks thus leading to a very small contribution from the "heavy" quarks when compared with the contribution coming from the "light" quarks.

In Table [VI, we have presented results for the magnetic moments of the spin $\frac{3}{2}+\rightarrow \frac{1}{2}+$ transitions corresponding to the charmless decuplet to octet transitions $(10 \rightarrow 8)$, single charmed sextet to anti-triplet transitions $(6 \rightarrow \overline{3})$, single charmed sextet to sextet transitions $(6 \rightarrow 6)$ and double charmed triplet to triplet transitions $(3 \rightarrow 3)$ transitions. We have also presented the results for the spin $\frac{1}{2}^{+} \rightarrow \frac{1}{2}^{+}$transitions corresponding to the charmless octet to octet transitions $(8 \rightarrow 8)$ and single charmed anti-triplet to sextet transitions $(\overline{3} \rightarrow 6)$. Experimental data is available for only the low lying $8 \rightarrow 8$ transition $\left(\Sigma^{0} \rightarrow \Lambda+\gamma\right)$. Our prediction for this decay is $1.60(1.61 \pm 0.08[3])$. There is no experimental data available for any other charmed baryons transition magnetic moments as well as for the other low lying spin $\frac{3}{2}^{+} \rightarrow \frac{1}{2}^{+}$transitions so we have presented the predictions of LCQSR [16] and Lattice QCD [43], wherever the results are available. For the magnetic moment of the $(\Delta \rightarrow p+\gamma)$ transition, an empirical estimate can be made from the helicity amplitudes $A_{\frac{1}{2}}=-0.135 \pm 0.005 \mathrm{GeV}^{-\frac{1}{2}}$, and $A_{\frac{3}{2}}=-0.250 \pm 0.008 \mathrm{GeV}^{-\frac{1}{2}}$ [3] as inputs in the decay rate and the magnetic moment extracted is $\mu(\Delta p)=3.46 \pm 0.03 \mu_{N}$ [46]. The magnetic moment of $\mu(\Delta p)$ transition is a long standing problem and most of the approaches in literature underestimate it. Our predicted value $2.87 \mu_{N}$ is below the experimental results. The implications of $\chi \mathrm{CQM}$ and Cheng-Li mechanism perhaps can be substantiated by future measurements of $\mu(\Delta p)$.

Implications of configuration mixing, quark masses and confinement effects have also been investigated. In the spin $\frac{1}{2}+$ baryon magnetic moments, it is found that the inclusion of Cheng-Li mechanism predicts the results in the right direction even when configuration mixing is not included, however, the inclusion of confinement effects alongwith configuration mixing plays a crucial role in fitting the individual magnetic moments. Interestingly, we find that the masses $M_{u}=M_{d}=330 \mathrm{MeV}$, after corrections due to configuration mixing and confinement effects, provide the best fit for the magnetic moments. This implies a deeper significance for the $\chi \mathrm{CQM}$ coupling breaking and the quark masses parameters employed.

\section{SUMMARY AND CONCLUSION}

To summarize, in order to enlarge the scope of SU(4) chiral constituent quark model $(\chi \mathrm{CQM})$ and to estimate the phenomenological contribution of $c \bar{c}$ fluctuations, we have carried out a detailed analysis of the magnetic moments 
of the low lying and charmed spin $\frac{1}{2}{ }^{+}$and spin $\frac{3}{2}^{+}$baryons as well as of their transitions. Using the generally accepted values of the quark masses, the parameters of $\chi \mathrm{CQM}$ have been fixed from the latest data pertaining to $\bar{u}-\bar{d}$ asymmetry and spin polarization functions, the explicit contributions coming from the valence quarks, the "quark sea" contribution as well as its orbital angular momentum through the generalized Cheng-Li mechanism have been calculated.

For the low lying $\frac{1}{2}^{+}$and spin $\frac{3}{2}^{+}$baryons where experimental data is available, the $\chi$ CQM predictions not only give a satisfactory fit but also show improvement over the other models. In particular, for the case of $\mu(p), \mu\left(\Sigma^{+}\right), \mu\left(\Xi^{0}\right)$, $\mu(\Lambda)$, violation of Coleman-Glashow sum rule for the spin $\frac{1}{2}^{+}$baryons and $\mu\left(\Delta^{+}\right), \mu\left(\Omega^{-}\right)$for the spin $\frac{3}{2}{ }^{+}$baryons, we are able to achieve an excellent agreement with data. For all the other low lying octet and decuplet baryons our predictions are within $10 \%$ of the observed values. For the spin $\frac{1}{2}^{+}$and spin $\frac{3}{2}+$ charmed baryon magnetic moments, our results are very much in agreement with recent theoretical estimates. It is observed that the orbital part contributes with the same sign as valence quark distribution, while the sea part contribute with the opposite sign. Further, for the cases where "light" quarks dominate in the valence structure, the resultant sea and orbital contributions are found to be fairly significant as compared to the valence contributions. On the other hand, when there is an excess of "heavy" quarks, the contribution of the "quark sea" is almost negligible, for example, $\mu\left(\Omega_{c}^{0}\right)$, $\mu\left(\Lambda_{c}^{+}\right), \mu\left(\Xi_{c}^{+}\right), \mu\left(\Xi_{c}^{0}\right), \mu\left(\Omega_{c c}^{+}\right), \mu\left(\Omega^{-}\right), \mu\left(\Omega_{c}^{* 0}\right), \mu\left(\Omega_{c c}^{*+}\right)$ and $\mu\left(\Omega_{c c c}^{*++}\right)$. However, it is interesting that the sea and orbital parts cancel in the right direction to give the correct magnitude of the total magnetic moment.

The implications of such a model have also been been studied for the case of low lying spin $\frac{3}{2}{ }^{+} \rightarrow \frac{1}{2}^{+}$transition magnetic moments as well as for the $\frac{1}{2}^{+} \rightarrow \frac{1}{2}^{+}$transitions involving charmed baryons. In this case also, the contribution of orbital angular momentum is found to be as important as that of the spin polarization of the $q \bar{q}$ pairs. Implications of configuration mixing and quark masses have also been investigated. Interestingly, we find that generalized Cheng-Li mechanism coupled with corrections due to configuration mixing and confinement effects, provide the best fit for the magnetic moments. This suggests that constituent quarks and weakly interacting Goldstone bosons provide the appropriate degree of freedom in the nonperturbative regime of QCD. This fact can perhaps can be substantiated by a measurement of the magnetic moments of charmed baryons. Several groups BTeV, SELEX Collaboration are contemplating the possibility of performing it in the near future.

\section{ACKNOWLEDGMENTS}

H.D. would like to thank Department of Science and Technology, Government of India, for financial support.

\section{Appendix A: The wave function convention for the baryon}

The total wave function for the three quark system made from any of the $u, d, s$ or $c$ quarks is given as $\mid S U(8) \otimes$ $O(3)\rangle=\varphi \chi \psi$, where $\varphi$ is a flavor wave function, $\chi$ is a spin wave function and $\psi$ is a spatial wave function. The $S U(8)$ multiplet is decomposed into $S U(4) \otimes S U(2)$ flavor and spin multiplets, respectively. The multiplet numerology for the subset of baryons belonging to $\mathrm{SU}(4)$ flavor multiplets, is $4 \times 4 \times 4=20_{S}+20_{M}+20_{M}+\overline{4}$, where the symmetry 20 -plet consists of $10+6+3+1$ and the mixed symmetry 20 -plet consists of $8+6+\overline{3}+3$ baryons flavor states. For the details of the definition of spatial part of the wave function $\left(\psi^{s}, \psi^{\prime}, \psi^{\prime \prime}\right)$ represented by the $O(3)$, we refer the reader to reference [47].

In order to understand the structure of charmed baryon wave functions and sign conventions used in this work, we present here the $S U(4) \otimes S U(2)$ content of the $S U(8)$ multiplet which is given as

$$
\begin{aligned}
120 & \supset{ }^{4} 20_{S}+{ }^{2} 20_{M}, \\
168 & \supset{ }^{2} 20_{S}+{ }^{4} 20_{M}+{ }^{2} 20_{M}+{ }^{2} \overline{4}, \\
56 & \supset{ }^{2} \overline{4}+{ }^{2} 20_{M} .
\end{aligned}
$$

The $S U(8) \otimes O(3)$ wave functions for the spin $\frac{1}{2}^{+}$and $\frac{3}{2}^{+}$baryons are respectively,

$$
\begin{aligned}
& |B\rangle \simeq\left|120,{ }^{2} 20_{M}\right\rangle_{N=0}=\frac{1}{\sqrt{2}}\left(\chi^{\prime} \varphi^{\prime}+\chi^{\prime \prime} \varphi^{\prime \prime}\right) \psi^{s}\left(0^{+}\right), \\
& \left|B^{*}\right\rangle \simeq\left|120,{ }^{4} 20_{S}\right\rangle_{N=0}=\chi^{s} \varphi^{s} \psi^{s}\left(0^{+}\right) .
\end{aligned}
$$

To incorporate the effect of configuration mixing generated by the spin-spin interactions [23, 30, 33] which has been shown to improve the prediction of the $\chi \mathrm{CQM}$, the complete wave function for the spin $\frac{1}{2}{ }^{+}$baryons can be expressed as

$$
|B\rangle=\cos \phi\left|120,{ }^{2} 20_{M}\right\rangle_{N=0}+\sin \phi\left|168,{ }^{2} 20_{M}\right\rangle_{N=2}
$$


where $\left|168,{ }^{2} 20_{M}\right\rangle_{N=2}=\frac{1}{2}\left(\left(\varphi^{\prime} \chi^{\prime \prime}+\varphi^{\prime \prime} \chi^{\prime}\right) \psi^{\prime}\left(0^{+}\right)+\left(\varphi^{\prime} \chi^{\prime}-\varphi^{\prime \prime} \psi^{\prime \prime}\right) \psi^{\prime \prime}\left(0^{+}\right)\right)$. The explicit flavor wave functions for the spin $\frac{1}{2}+$ baryons are as follows

\begin{tabular}{cccc}
\hline & Baryon & $\varphi^{\prime}$ & $\varphi^{\prime \prime}$ \\
\hline$(8,0)$ & $p$ & $\frac{1}{\sqrt{2}}(u d u-d u u)$ & $\frac{1}{\sqrt{6}}(2 u u d-u d u-d u u)$ \\
& $n$ & $\frac{1}{\sqrt{2}}(u d d-d u d)$ & $\frac{1}{\sqrt{6}}(d u d+u d d-2 d d u)$ \\
& $\Sigma^{+}$ & $\frac{1}{\sqrt{2}}(u s u-s u u)$ & $\frac{1}{\sqrt{6}}(2 u u s-s u u-u s u)$ \\
& $\Sigma^{0}$ & $\frac{1}{2}(s u d+s d u-u s d-d s u)$ & $\frac{1}{2 \sqrt{3}}(s d u+d s u+s u d+u s d-2 u d s-2 d u s)$ \\
& $\Sigma^{-}$ & $\frac{1}{\sqrt{2}}(s d d-d s d)$ & $\frac{1}{\sqrt{6}}(2 d d s-s d d-d s d)$ \\
& $\Lambda$ & $\frac{1}{2 \sqrt{3}}(2 u d s-2 d u s+s d u-d s u+u s d-s u d)$ & $\frac{1}{2}(s u d+u s d-s d u-d s u)$ \\
& $\Xi^{0}$ & $\frac{1}{\sqrt{2}}(s u s-u s s)$ & $\frac{1}{\sqrt{6}}(s u s+u s s-2 s s u)$ \\
& $\Xi^{-}$ & $\frac{1}{\sqrt{2}}(s d s-d s s)$ & $\frac{1}{\sqrt{6}}(s d s+d s s-2 s s d)$ \\
\hline$(6,1)$ & $\Sigma_{c}^{++}$ & $\frac{1}{\sqrt{2}}(c u u-u c u)$ & $\frac{1}{\sqrt{6}}(c u u+u c u-2 u u c)$ \\
& $\Sigma_{c}^{+}$ & $\frac{1}{2}(c u d+c d u-u c d-d c u)$ & $\frac{1}{\sqrt{6}}(c d d+d c d-2 d d c)$ \\
& $\Sigma_{c}^{0}$ & $\frac{1}{\sqrt{2}}(c d d-d c d)$ & $\frac{1}{2 \sqrt{3}}(d c u+c d u+u c d+c u d-2 u d c-2 d u c)$ \\
& $\Xi_{c}^{\prime}$ & $\frac{1}{2}(c u s+c s u-u c s-s c u)$ & $\frac{1}{2 \sqrt{3}}(d c s+c d s+s c d+c s d-2 d s c-2 s d c)$ \\
& $\Xi_{c}^{\prime}$ & $\frac{1}{2}(c d s+c s d-d c s-s c d)$ & $\frac{1}{\sqrt{6}}(s c s+c s s-2 s s c)$ \\
& $\Omega_{c}^{0}$ & $\frac{1}{\sqrt{2}}(c s s-s c s)$ & $\frac{1}{2}(u c d+c u d-d c u-c d u)$ \\
\hline$(\overline{3}, 1)$ & $\Lambda_{c}^{+}$ & $\frac{1}{2 \sqrt{3}}(2 u d c-2 d u c+c d u-d c u+u c d-c u d)$ & $\frac{1}{2}(u c s+c u s-s c u-c s u)$ \\
& $\Xi_{c}^{+}$ & $\frac{1}{2 \sqrt{3}}(2 u s c-2 s u c+c s u-s c u+u c s-c u s)$ & $\frac{1}{2}(d c s+c d s-s c d-c s d)$ \\
& $\Xi_{c}^{0}$ & $\frac{1}{2 \sqrt{3}}(2 d s c-2 s d c+c s d-s c d+d c s-c d s)$ & $\frac{1}{\sqrt{6}}(u c c+c u c-2 c c u)$ \\
\hline$(3,2)$ & $\Xi_{c c}^{++}$ & $\frac{1}{\sqrt{6}}(d c c+c d c-2 c c d)$ \\
& $\Xi_{c c}^{+}$ & $\frac{1}{\sqrt{2}}(d c c-c d c)$ & $\frac{1}{\sqrt{6}}(s c c+c s c-2 c c s)$ \\
\hline$\Omega_{c c}^{+}$ & $\frac{1}{\sqrt{2}}(s c c-c s c)$ & \\
\hline
\end{tabular}


For the spin $\frac{3}{2}+$ baryons, the flavor wavefunctions are

\begin{tabular}{|c|c|c|}
\hline & Baryon & $\varphi^{s}$ \\
\hline \multirow[t]{10}{*}{$(10,0)$} & $\Delta^{++}$ & uuu \\
\hline & $\Delta^{+}$ & $\frac{1}{\sqrt{3}}(u u d+u d u+d u u)$ \\
\hline & $\Delta^{0}$ & $\frac{1}{\sqrt{3}}(u d d+d d u+d u d)$ \\
\hline & $\Delta^{-}$ & $d d d$ \\
\hline & $\Sigma^{*+}$ & $\frac{1}{\sqrt{3}}(u u s+s u u+u s u)$ \\
\hline & $\Sigma^{*-}$ & $\frac{1}{\sqrt{3}}(d d s+d s d+s d d)$ \\
\hline & $\Sigma^{* 0}$ & $\frac{1}{\sqrt{6}}(s d u+s u d+u s d+d s u+d u s+u d s)$ \\
\hline & $\Xi^{* 0}$ & $\frac{1}{\sqrt{3}}(s s u+s u s+u s s)$ \\
\hline & $\Xi^{*-}$ & $\frac{1}{\sqrt{3}}(s s d+s d s+d s s)$ \\
\hline & $\Omega^{-}$ & sss \\
\hline \multirow[t]{6}{*}{$(6,1)$} & $\Sigma_{c}^{*++}$ & $\frac{1}{\sqrt{3}}(u u c+u c u+c u u)$ \\
\hline & $\Sigma_{c}^{*+}$ & $\frac{1}{\sqrt{6}}(u d c+d c u+c u d+c d u+d u c+u c d)$ \\
\hline & $\Sigma_{c}^{* 0}$ & $\frac{1}{\sqrt{3}}(d d c+d c d+c d d)$ \\
\hline & $\Xi_{c}^{*+}$ & $\frac{1}{\sqrt{6}}(u s c+s c u+c u s+c s u+s u c+u c s)$ \\
\hline & $\Xi_{c}^{* 0}$ & $\frac{1}{\sqrt{6}}(d s c+s c d+c d s+c s d+d s c+s c d)$ \\
\hline & $\Omega_{c}^{* 0}$ & $\frac{1}{\sqrt{3}}(s s c+s c s+c s s)$ \\
\hline \multirow[t]{3}{*}{$(3,2)$} & $\Xi_{c c}^{*++}$ & $\frac{1}{\sqrt{3}}(u c c+c u c+c c u)$ \\
\hline & $\Xi_{c c}^{*+}$ & $\frac{1}{\sqrt{3}}(d c c+c d c+c c d)$ \\
\hline & $\Omega_{c c}^{*+}$ & $\frac{1}{\sqrt{3}}(s c c+c s c+c c s)$ \\
\hline$(1,3)$ & $\Omega_{c c c}^{*+}$ & $c c c$ \\
\hline
\end{tabular}

We have used the convention $\chi=\chi_{S_{z}}^{\sigma}$ for the spin wave functions, where $S_{z}$ is the third component of the spin and $\sigma$ represents the symmetry state

$$
\chi_{\frac{3}{2}}^{s}=\uparrow \uparrow \uparrow, \quad \chi_{\frac{1}{2}}^{\prime}=\frac{1}{\sqrt{2}}(\uparrow \downarrow \uparrow-\downarrow \uparrow \uparrow), \quad \chi_{\frac{1}{2}}^{\prime \prime}=\frac{1}{\sqrt{6}}(2 \uparrow \uparrow \downarrow-\uparrow \downarrow \uparrow-\downarrow \uparrow \uparrow) .
$$

Other values of $S_{z}$ are obtained by applying the lowering the operator in spin space and normalizing to unity.

[1] S.J. Brodsky, P. Hoyer, C. Peterson, and N. Sakai, Phys. Lett. B 93, 451 (1980); S.J. Brodsky, C. Peterson, and N. Sakai, Phys. Rev. D 23, 2745 (1981).

[2] H. Garcilazo, J. Vijande, and A. Valcarce, J. Phys. G 34, 961 (2007).

[3] C. Amsler et al., Phys. Lett. B 667, 1 (2008).

[4] A.L. Choudhury and V. Joshi, Phys. Rev. D 13, 3115 (1976); ibid. 3120 (1976); D.B. Lichtenberg, Phys. Rev. D 15, 345 (1977); R.J. Johnson and M. Shah-Jahan, ibid. 1400 (1977).

[5] S.N. Jena and D.P. Rath, Phys. Rev. D 34, 196 (1986).

[6] L.Ya. Glozman and D.O. Riska, Nucl. Phys. A 603, 326 (1996); Erratum-ibid. 620, 510 (1997).

[7] B. Julia Diaz and D.O. Riska, Nucl. Phys. A 739, 69 (2004).

[8] Yongseok Oh and Byung-Yoon Park, Mod. Phys. Lett. A 11, 653 (1996).

[9] S. Scholl and H. Weigel, Nucl. Phys. A 735, 163 (2004).

[10] A. Faessler et al., Phys. Rev. D 73, 094013 (2006).

[11] S. Kumar, R. Dhir, and R.C. Verma, Jol. Phys. G 31, 141 (2005); A. Majethiya, B. Patel, and P.C. Vinodkumar, Eur. Phys. J.A 38, 307 (2008); B. Patel, A.K. Rai, and P.C. Vinodkumar, Jol. of Phys. G 35, 065001 (2008); R. Dhir and R.C. Verma, Eur. Phys. J. A 42, 243 (2009).

[12] F.X. Lee, Phys. Rev. D 57, 1801 (1998); Lai Wang and F.X. Lee, Phys. Rev. D 78, 013003 (2008).

[13] Shi-lin Zhu, W-Y.P. Hwang, and Ze-sen Yang, Phys. Rev. D 56, 7273 (1997).

[14] T.M. Aliev, A. Ozpineci, and M. Savci, Phys. Rev. D 66, 016002 (2002); ibid. D 67, 039901 (2003); T.M. Aliev, K. Azizi and A. Ozpineci, Phys. Rev. D 77, 114006 (2008). 
[15] T.M. Aliev, A. Ozpineci, and M. Savci, Phys. Rev. D 62, 053012 (2000).

[16] T.M. Aliev, A. Ozpineci, and M. Savci, Phys. Lett. B 516, 299 (2001); Phys. Rev. D 65, 096004 (2002); T.M. Aliev, K. Azizi, and A. Ozpineci, ibid. 79, 056005 (2009).

[17] T. Hatsuda, and T. Kunihiro, Phys. Rep. 247, 221 (1994); F.S. Navarra et al., Phys. Rev. D 54, 842 (1996); A. Blotz and E. Shuryak, Phys. Lett. B 439, 415 (1998); M.V. Polyakov, A. Schafer, and O.V. Teryaev, Phys. Rev. D 60, 051502(R) (1999); M. Franz, M.V. Polyakov, and K. Goeke, Phys. Rev. D 62, 074024 (2000).

[18] S. Weinberg, Physica A 96, 327(1979); A. Manohar and H. Georgi, Nucl. Phys. B 234, 189 (1984).

[19] E.J. Eichten, I. Hinchliffe, and C. Quigg, Phys. Rev. D 45, 2269 (1992).

[20] T.P. Cheng and Ling Fong Li, Phys. Rev. Lett. 74, 2872 (1995); Phys. Rev. D 57, 344 (1998); hep-ph/9709293

[21] A. De Rujula, H. Georgi, and S.L. Glashow, Phys. Rev. D 12, 147 (1975).

[22] N. Isgur, G. Karl, and R. Koniuk, Phys. Rev. Lett. 41, 1269 (1978); R. Koniuk and N. Isgur, Phys. Rev. D 21, 1868 (1980); N. Isgur and G. Karl, Phys. Rev. D 21, 3175 (1980); N. Isgur et al., Phys. Rev. D 35, 1665 (1987).

[23] H. Dahiya and M. Gupta, Phys. Rev. D 64, 014013 (2001); H. Dahiya and M. Gupta, Int. Jol. of Mod. Phys. A, Vol. 19, No. 29, 5027 (2004); H. Dahiya, M. Gupta, and J.M.S. Rana, Int. Jol. of Mod. Phys. A, Vol. 21, No. 21, 4255 (2006); H. Dahiya and M. Gupta, Eur. Phys. J. C 52, 571 (2007).

[24] X. Song, J.S. McCarthy, and H.J. Weber, Phys. Rev. D 55, 2624 (1997); X. Song, Phys. Rev. D 57, 4114 (1998).

[25] H. Dahiya and M. Gupta, Phys. Rev. D 78, 014001 (2008).

[26] N. Sharma, H. Dahiya, P.K. Chatley, and M. Gupta, Phys. Rev. D 79, 077503 (2009).

[27] S. Coleman and S.L. Glashow, Phys. Rev. Lett. 6, 423(1961).

[28] T.P. Cheng and Ling Fong Li, Phys. Rev. Lett. 80, 2789 (1998).

[29] J. Linde, T. Ohlsson, and H. Snellman, Phys. Rev. D 57, 452 (1998); ibid. 57, 5916 (1998).

[30] H. Dahiya and M. Gupta, Phys. Rev. D 66, 051501(R) (2002); H. Dahiya and M. Gupta, Phys. Rev. D 67, 114015 (2003).

[31] T.P. Cheng and Ling Fong Li, hep-ph/9811279.

[32] X. Song, Phys. Rev. D 65, 114022 (2002); X. Song, Int. Jol. of Mod. Phys. A 18, 11501 (2003).

[33] H. Dahiya and M. Gupta, Phys. Rev. D 67, 074001 (2003).

[34] J. Ashman et al. (European Muon Collaboration), Phys. Lett. B 206, 364 (1988); Nucl. Phys. 328, 1 (1989).

[35] B. Adeva et al. (SMC Collaboration), Phys. Lett. B 302, 533 (1993); D. Adams et al., Phys. Rev. D 56, 5330 (1997).

[36] J. Ellis and M. Karliner, Phys. Lett. B 313, 131 (1993); ibid. 41, 397 (1995).

[37] P. Amaudruz et al. (New Muon Collaboration), Phys. Rev. Lett. 66, 2712 (1991); M. Arneodo et al., Phys. Rev. D 50, R1 (1994).

[38] E.A. Hawker et al. (E866/NuSea Collaboration), Phys. Rev. Lett. 80, 3715 (1998); J.C. Peng et al., Phys. Rev. D 58, 092004 (1998); R.S. Towell et al., Phys. Rev. D 64, 052002 (2001).

[39] A. Le Yaouanc, L. Oliver, O. Pene, and J.C. Raynal, Phys. Rev. D 12, 2137 (1975); ibid. 15, 844 (1977); M. Gupta and A.N. Mitra, Phys. Rev. D 18, 1585 (1978).

[40] V. Elias, Mong Tong, and M.D. Scadron, Phys. Rev. D 40, 3670 (1989); Duane A. Dicus, Djordge Minic, Ubirajara van Klock, and Roberto Vega, Phys. Lett. 284, 384 (1992); Y.B. Donget al., J. Phys. G 25, 1115 (1999).

[41] Ikuo S. Sogami and Noboru Ohýamaguchi, Phys. Rev. Lett. 54, 2295 (1985); Kuang-Ta Chao, Phys. Rev. D 41, 920 (1990); M. Gupta, J. Phys. G 16, L213 (1990).

[42] B.O. Kerbikov and Yu. A. Simonov, Phys. Rev. D 62, 093016 (2000); B.O. Kerbikov, Phys. Atom. Nucl. 64, 1856 (2001).

[43] D.B. Leinweber, R.M. Woloshyn, and T. Draper, Phys. Rev. D 43, 1659 (1991); D.B. Leinweber, Phys. Rev. D 45, 252 (1992); I.C. Cloet, D.B. Leinweber, and A.W. Thomas, Phys. Lett. B 563157 (2003); D.B. Leinweber, T. Draper, and R.M. Woloshyn, Phys. Rev. D 46, 3067 (1992).

[44] M. Kotulla et al., Phys. Rev. Lett. 89, 272001 (2002); M. Kotulla, Prog. Part. Nucl. Phys. 61, 147 (2008).

[45] H.T. Diehl et al., Phys. Rev. Lett. 67, 804 (1991).

[46] L. Tiator et al., Nucl. Phys. A 689, 205 (2001).

[47] A. Le Yaouanc et al., Hadron Transitions in the Quark Model, Gordon and Breach (1988). 


\begin{tabular}{|c|c|c|}
\hline Baryon & & Valence and sea contribution to the magnetic moments \\
\hline \multirow[t]{2}{*}{$p$} & $\mu_{\text {val }}$ & $\cos ^{2} \phi\left[\frac{4}{3} \mu_{u}-\frac{1}{3} \mu_{d}\right]+\sin ^{2} \phi\left[\frac{2}{3} \mu_{u}+\frac{1}{3} \mu_{d}\right]$ \\
\hline & & $\begin{aligned} & \cos ^{2} \phi\left.-\frac{a}{3}\left(7+4 \alpha^{2}+\frac{4}{3} \beta^{2}+\frac{\zeta^{2}}{6}+\frac{17}{4} \gamma^{2}\right) \mu_{u}-\frac{a}{3}\left(2-\alpha^{2}-\frac{\beta^{2}}{3}-\frac{\zeta^{2}}{24}-\frac{17}{16} \gamma^{2}\right) \mu_{d}-\left(a \alpha^{2}\right) \mu_{s}-\left(a \gamma^{2}\right) \mu_{c}\right] \\
&+\sin ^{2} \phi\left[-\frac{a}{3}\left(5+2 \alpha^{2}+\frac{2}{3} \beta^{2}+\frac{\zeta^{2}}{12}+\frac{17}{8} \gamma^{2}\right) \mu_{u}-\frac{a}{3}\left(4+\alpha^{2}+\frac{\beta^{2}}{3}+\frac{\zeta^{2}}{24}+\frac{17}{16} \gamma^{2}\right) \mu_{d}-\left(a \alpha^{2}\right) \mu_{s}-\left(a \gamma^{2}\right) \mu_{c}\right]\end{aligned}$ \\
\hline \multirow[t]{2}{*}{$\Sigma^{+}$} & $\mu_{\mathrm{val}}$ & $\cos ^{2} \phi\left[\frac{4}{3} \mu_{u}-\frac{1}{3} \mu_{s}\right]+\sin ^{2} \phi\left[\frac{2}{3} \mu_{u}+\frac{1}{3} \mu_{s}\right]$ \\
\hline & & $\begin{aligned} & \cos ^{2} \phi\left.-\frac{a}{3}\left(8+3 \alpha^{2}+\frac{4}{3} \beta^{2}+\frac{\zeta^{2}}{6}+\frac{17}{4} \gamma^{2}\right) \mu_{u}-\frac{a}{3}\left(4-\alpha^{2}\right) \mu_{d}-\frac{a}{3}\left(2 \alpha^{2}-\frac{4}{3} \beta^{2}-\frac{\zeta^{2}}{24}-\frac{17}{16} \gamma^{2}\right) \mu_{s}-\left(a \gamma^{2}\right) \mu_{c}\right] \\
&+\sin ^{2} \phi\left[-\frac{a}{3}\left(4+6 \alpha^{2}+\frac{2}{3} \beta^{2}+\frac{\zeta^{2}}{12}+\frac{17}{8} \gamma^{2}\right) \mu_{u}-\frac{a}{3}\left(2+\alpha^{2}\right) \mu_{d}-\frac{a}{3}\left(4 \alpha^{2}+\frac{4}{3} \beta^{2}+\frac{\zeta^{2}}{24}+\frac{17}{16} \gamma^{2}\right) \mu_{s}-\left(a \gamma^{2}\right) \mu_{c}\right]\end{aligned}$ \\
\hline \multirow[t]{2}{*}{$\Sigma^{0}$} & $\mu_{\mathrm{val}}$ & $\cos ^{2} \phi\left[\frac{2}{3} \mu_{u}+\frac{2}{3} \mu_{d}-\frac{1}{3} \mu_{s}\right]+\sin ^{2} \phi\left[\frac{1}{3} \mu_{u}+\frac{1}{3} \mu_{d}+\frac{1}{3} \mu_{s}\right]$ \\
\hline & & $\begin{array}{l}\cos ^{2} \phi\left[-\frac{a}{3}\left(6+\alpha^{2}+\frac{2}{3} \beta^{2}+\frac{\zeta^{2}}{12}+\frac{17}{8} \gamma^{2}\right) \mu_{u}-\frac{a}{3}\left(6+\alpha^{2}+\frac{2}{3} \beta^{2}+\frac{\zeta^{2}}{12}+\frac{17}{8} \gamma^{2}\right) \mu_{d}-\frac{a}{3}\left(+2 \alpha^{2}-\frac{4}{3} \beta^{2}-\frac{\zeta^{2}}{24}-\frac{17}{16} \gamma^{2}\right) \mu_{s}-\left(a \gamma^{2}\right) \mu_{c}\right. \\
+\sin ^{2} \phi\left[-\frac{a}{3}\left(3+2 \alpha^{2}+\frac{\beta^{2}}{3}+\frac{\zeta^{2}}{24}+\frac{17}{16} \gamma^{2}\right) \mu_{u}-\frac{a}{3}\left(3+2 \alpha^{2}+\frac{\beta^{2}}{3}+\frac{\zeta^{2}}{24}+\frac{17}{16} \gamma^{2}\right) \mu_{d}-\frac{a}{3}\left(4 \alpha^{2}+\frac{4}{3} \beta^{2}+\frac{\zeta^{2}}{24}+\frac{17}{16} \gamma^{2}\right) \mu_{s}-\left(a \gamma^{2}\right) \mu_{c}\right.\end{array}$ \\
\hline \multirow[t]{2}{*}{$\Xi^{0}$} & $\mu_{\mathrm{val}}$ & $\cos ^{2} \phi\left[-\frac{1}{3} \mu_{u}+\frac{4}{3} \mu_{s}\right]+\sin ^{2} \phi\left[\frac{1}{3} \mu_{u}+\frac{2}{3} \mu_{s}\right]$ \\
\hline & & $\begin{aligned} & \cos ^{2} \phi\left.-\frac{a}{3}\left(-2+3 \alpha^{2}-\frac{\beta^{2}}{3}-\frac{\zeta^{2}}{24}-\frac{17}{16} \gamma^{2}\right) \mu_{u}-\frac{a}{3}\left(-1+4 \alpha^{2}\right) \mu_{d}-\frac{a}{3}\left(7 \alpha^{2}+\frac{16}{3} \beta^{2}+\frac{\zeta^{2}}{6}+\frac{17}{4} \gamma^{2}\right) \mu_{s}-\left(a \gamma^{2}\right) \mu_{c}\right] \\
&+\sin ^{2} \phi\left[-\frac{a}{3}\left(2+3 \alpha^{2}+\frac{\beta^{2}}{3}+\frac{\zeta^{2}}{24}+\frac{17}{16} \gamma^{2}\right) \mu_{u}-\frac{a}{3}\left(1+2 \alpha^{2}\right) \mu_{d}-\frac{a}{3}\left(5 \alpha^{2}+\frac{8}{3} \beta^{2}+\frac{\zeta^{2}}{12}+\frac{17}{8} \gamma^{2}\right) \mu_{s}-\left(a \gamma^{2}\right) \mu_{c}\right]\end{aligned}$ \\
\hline \multirow[t]{2}{*}{$\Lambda$} & $\mu_{\mathrm{val}}$ & $\cos ^{2} \phi\left[\mu_{s}\right]+\sin ^{2} \phi\left[\frac{1}{3} \mu_{u}+\frac{1}{3} \mu_{d}+\frac{1}{3} \mu_{s}\right]$ \\
\hline & $\mu_{\text {sea }}$ & $\begin{array}{c}\cos ^{2} \phi\left[-\left(a \alpha^{2}\right) \mu_{u}-\left(a \alpha^{2}\right) \mu_{d}-a\left(2 \alpha^{2}+\frac{4}{3} \beta^{2}+\frac{\zeta^{2}}{24}+\frac{17}{16} \gamma^{2}\right) \mu_{s}-\left(a \gamma^{2}\right) \mu_{c}\right] \\
+\sin ^{2} \phi\left[-\frac{a}{3}\left(2+2 \alpha^{2}+\frac{\beta^{2}}{3}+\frac{\zeta^{2}}{24}+\frac{17}{16} \gamma^{2}\right) \mu_{u}-\frac{a}{3}\left(2+2 \alpha^{2}+\frac{\beta^{2}}{3}+\frac{\zeta^{2}}{24}+\frac{17}{16} \gamma^{2}\right) \mu_{d}-\frac{a}{3}\left(4 \alpha^{2}+\frac{4}{3} \beta^{2}+\frac{\zeta^{2}}{24}+\frac{17}{16} \gamma^{2}\right) \mu_{s}-\left(a \gamma^{2}\right) \mu_{c}\right]\end{array}$ \\
\hline \multirow[t]{2}{*}{$\Sigma_{c}^{++}$} & $\mu_{\mathrm{val}}$ & $\cos ^{2} \phi\left[\frac{4}{3} \mu_{u}-\frac{1}{3} \mu_{c}\right]+\sin ^{2} \phi\left[\frac{2}{3} \mu_{u}+\frac{1}{3} \mu_{c}\right]$ \\
\hline & & $\begin{array}{c}\cos ^{2} \phi\left[-\frac{a}{3}\left(8+4 \alpha^{2}+\frac{4}{3} \beta^{2}+\frac{\zeta^{2}}{6}+\frac{13}{4} \gamma^{2}\right) \mu_{u}-\frac{a}{3}\left(4-\gamma^{2}\right) \mu_{d}-\frac{a}{3}\left(4 \alpha^{2}-\gamma^{2}\right) \mu_{s}+\frac{a}{24}\left(3 \zeta^{2}+\gamma^{2}\right) \mu_{c}\right] \\
\sin ^{2} \phi\left[-\frac{a}{3}\left(4+2 \alpha^{2}+\frac{2}{3} \beta^{2}+\frac{\zeta^{2}}{12}+\frac{25}{8} \gamma^{2}\right) \mu_{u}-\frac{a}{3}\left(2+\gamma^{2}\right) \mu_{d}-\frac{a}{3}\left(2 \alpha^{2}+\gamma^{2}\right) \mu_{s}-\frac{a}{24}\left(3 \zeta^{2}+49 \gamma^{2}\right) \mu_{c}\right]\end{array}$ \\
\hline \multirow[t]{2}{*}{$\Sigma_{c}^{+}$} & $\mu_{\mathrm{val}}$ & $\cos ^{2} \phi\left[\frac{2}{3} \mu_{u}+\frac{2}{3} \mu_{d}-\frac{1}{3} \mu_{c}\right]+\sin ^{2} \phi\left[\frac{1}{3} \mu_{u}+\frac{1}{3} \mu_{d}+\frac{1}{3} \mu_{c}\right]$ \\
\hline & $\mu_{\text {sea }}$ & \begin{tabular}{c|c|c|}
$\cos ^{2} \phi$ & $-\frac{a}{3}\left(6+2 \alpha^{2}+\frac{2}{3} \beta^{2}+\frac{\zeta^{2}}{12}+\frac{9}{8} \gamma^{2}\right) \mu_{u}-\frac{a}{3}\left(6+2 \alpha^{2}+\frac{2}{3} \beta^{2}+\frac{\zeta^{2}}{12}+\frac{9}{8} \gamma^{2}\right) \mu_{d}-\frac{a}{3}\left(4 \alpha^{2}-\gamma^{2}\right) \mu_{s}+\frac{a}{24}\left(3 \zeta^{2}+\gamma^{2}\right) \mu_{c}$ \\
$\sin ^{2} \phi$ & $\left.-\frac{a}{3}\left(3+\alpha^{2}+\frac{\beta^{2}}{3}+\frac{\zeta^{2}}{24}+\frac{33}{16} \gamma^{2}\right) \mu_{u}-\frac{a}{3}\left(3+\alpha^{2}+\frac{\beta^{2}}{3}+\frac{\zeta^{2}}{24}+\frac{33}{16} \gamma^{2}\right) \mu_{d}-\frac{a}{3}\left(2 \alpha^{2}+\gamma^{2}\right) \mu_{s}-\frac{a}{24}\left(3 \zeta^{2}+49 \gamma^{2}\right) \mu_{c}\right]$
\end{tabular} \\
\hline \multirow[t]{2}{*}{$\Omega_{c}^{0}$} & $\mu_{\mathrm{val}}$ & $\cos ^{2} \phi\left[\frac{4}{3} \mu_{s}-\frac{1}{3} \mu_{c}\right]+\sin ^{2} \phi\left[\frac{2}{3} \mu_{s}+\frac{1}{3} \mu_{c}\right]$ \\
\hline & $\mu_{\text {sea }}$ & $\begin{array}{l}\cos ^{2} \phi\left[-\frac{a}{3}\left(4 \alpha^{2}-\gamma^{2}\right) \mu_{u}-\frac{a}{3}\left(4 \alpha^{2}-\gamma^{2}\right) \mu_{d}-\frac{a}{3}\left(8 \alpha^{2}+\frac{16}{3} \beta^{2}+\frac{\zeta^{2}}{6}+\frac{13}{4} \gamma^{2}\right) \mu_{s}+\frac{a}{24}\left(3 \zeta^{2}+\gamma^{2}\right) \mu_{c}\right] \\
\sin ^{2} \phi\left[-\frac{a}{3}\left(2 \alpha^{2}+\gamma^{2}\right) \mu_{u}-\frac{a}{3}\left(2 \alpha^{2}+\gamma^{2}\right) \mu_{d}-\frac{a}{3}\left(4 \alpha^{2}+\frac{8}{3} \beta^{2}+\frac{\zeta^{2}}{12}+\frac{33}{8} \gamma^{2}\right) \mu_{s}-\frac{a}{24}\left(3 \zeta^{2}+49 \gamma^{2}\right) \mu_{c}\right]\end{array}$ \\
\hline \multirow[t]{2}{*}{$\Lambda_{c}^{+}$} & $\mu_{\mathrm{val}}$ & $\cos ^{2} \phi\left[\mu_{c}\right]+\frac{1}{3} \sin ^{2} \phi\left[\mu_{u}+\mu_{d}+\mu_{c}\right]$ \\
\hline & $\mu_{\text {sea }}$ & $\begin{array}{c}\cos ^{2} \phi\left[-\left(a \gamma^{2}\right) \mu_{u}-\left(a \gamma^{2}\right) \mu_{d}-\left(a \gamma^{2}\right) \mu_{s}-\frac{3}{8} a\left(\zeta^{2}+11 \gamma^{2}\right) \mu_{c}\right] \\
+\sin ^{2} \phi\left[-\frac{a}{3}\left(3+\alpha^{2}+\frac{\beta^{2}}{3}+\frac{\zeta^{2}}{24}+\frac{33}{16} \gamma^{2}\right) \mu_{u}-\frac{a}{3}\left(3+\alpha^{2}+\frac{\beta^{2}}{3}+\frac{\zeta^{2}}{24}+\frac{33}{16} \gamma^{2}\right) \mu_{d}-\frac{a}{3}\left(2 \alpha^{2}+\gamma^{2}\right) \mu_{s}-\frac{a}{24}\left(3 \zeta^{2}+49 \gamma^{2}\right) \mu_{c}\right]\end{array}$ \\
\hline \multirow[t]{2}{*}{$\Xi_{c}^{+}$} & $\mu_{\mathrm{val}}$ & $\cos ^{2} \phi\left[\mu_{c}\right]+\frac{1}{3} \sin ^{2} \phi\left[\mu_{u}+\mu_{s}+\mu_{c}\right]$ \\
\hline & $\mu_{\text {sea }}$ & $\begin{array}{c}\cos ^{2} \phi\left[-\left(a \gamma^{2}\right) \mu_{u}-\left(a \gamma^{2}\right) \mu_{d}-\left(a \gamma^{2}\right) \mu_{s}-\frac{3}{8} a\left(\zeta^{2}+11 \gamma^{2}\right) \mu_{c}\right] \\
+\sin ^{2} \phi\left[-\frac{a}{3}\left(2+2 \alpha^{2}+\frac{\beta^{2}}{3}+\frac{\zeta^{2}}{24}+\frac{33}{16} \gamma^{2}\right) \mu_{u}-\frac{a}{3}\left(1+\alpha^{2}+\gamma^{2}\right) \mu_{d}-\frac{a}{3}\left(3 \alpha^{2}+\frac{4}{3} \beta^{2}+\frac{\zeta^{2}}{24}+\frac{33}{16} \gamma^{2}\right) \mu_{s}-\frac{a}{24}\left(3 \zeta^{2}+49 \gamma^{2}\right) \mu_{c}\right]\end{array}$ \\
\hline \multirow[t]{2}{*}{$\Omega_{c c}^{+}$} & $\mu_{\mathrm{val}}$ & $\cos ^{2} \phi\left[-\frac{1}{3} \mu_{s}+\frac{4}{3} \mu_{c}\right]+\sin ^{2} \phi\left[\frac{1}{3} \mu_{s}+\frac{2}{3} \mu_{c}\right]$ \\
\hline & $\mu_{\text {sea }}$ & \begin{tabular}{r|l}
$\cos ^{2} \phi$ & $\left.\frac{a}{3}\left(\alpha^{2}-4 \gamma^{2}\right) \mu_{u}+\frac{a}{3}\left(\alpha^{2}-4 \gamma^{2}\right) \mu_{d}+\frac{a}{3}\left(2 \alpha^{2}+\frac{4}{3} \beta^{2}+\frac{\zeta^{2}}{24}-\frac{47}{16} \gamma^{2}\right) \mu_{s}-\frac{a}{2}\left(\zeta^{2}+\frac{31}{3} \gamma^{2}\right) \mu_{c}\right]$ \\
$+\sin ^{2} \phi$ & {$\left[-\frac{a}{3}\left(\alpha^{2}+2 \gamma^{2}\right) \mu_{u}-\frac{a}{3}\left(\alpha^{2}+2 \gamma^{2}\right) \mu_{d}-\frac{a}{3}\left(2 \alpha^{2}+\frac{4}{3} \beta^{2}+\frac{\zeta^{2}}{24}+\frac{49}{16} \gamma^{2}\right) \mu_{s}-\frac{a}{4}\left(\zeta^{2}+\frac{37}{3} \gamma^{2}\right) \mu_{c}\right]$} \\
\end{tabular} \\
\hline
\end{tabular}

TABLE I: Valence and sea contribution of the charmed spin $\frac{1}{2}{ }^{+}$baryons in terms of $\chi$ CQM parameters and configuration mixing parameter $\phi$. The spin polarizations for the other baryons can be found from isospin symmetry. 


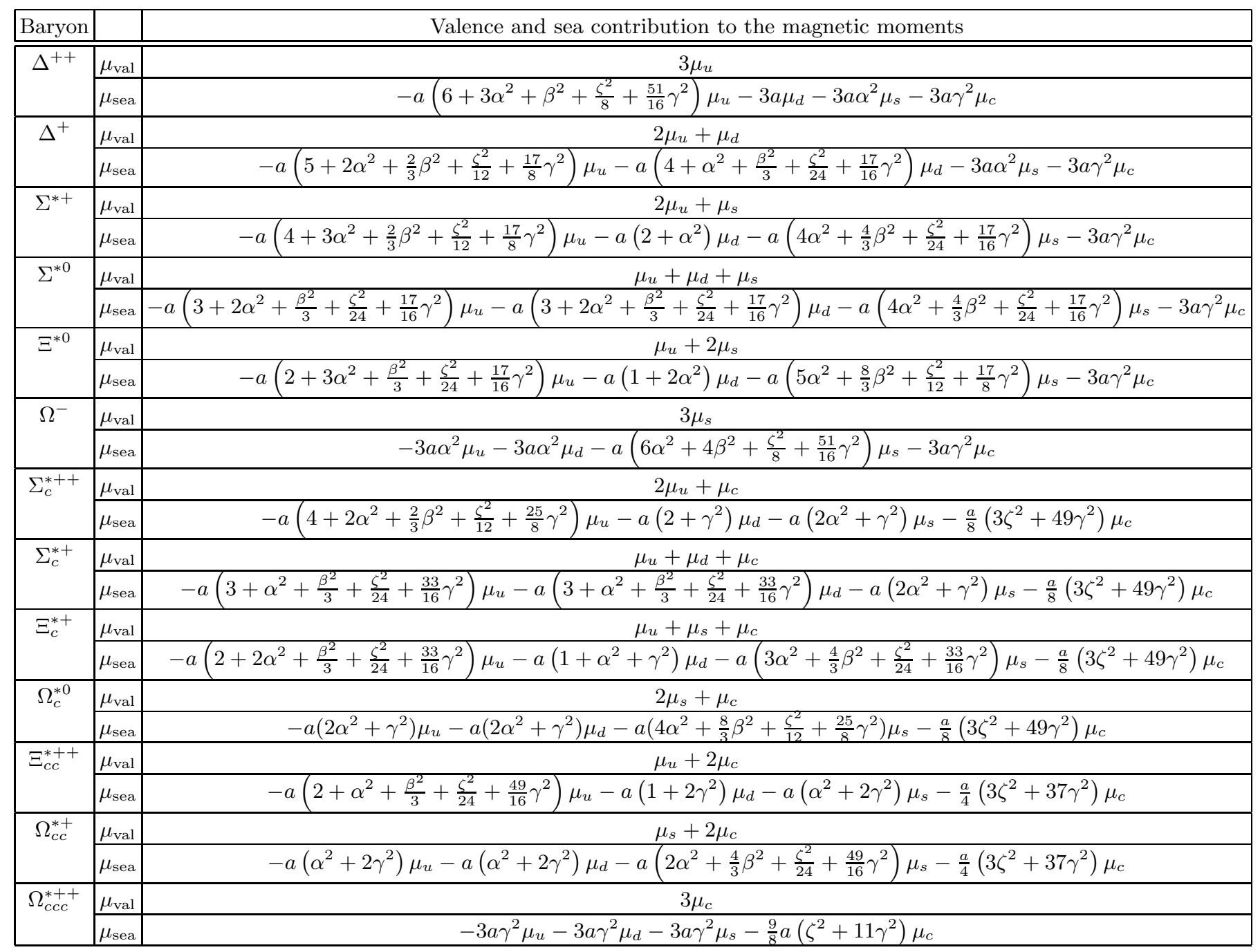

TABLE II: Valence and sea contributions of the low lying and charmed spin $\frac{3}{2}^{+}$baryons in terms of the $\chi \mathrm{CQM}$ parameters. The spin polarizations for the other baryons can be found from isospin symmetry. 


\begin{tabular}{|c|c|c|}
\hline Transition & & Valence and sea contribution to the transition magnetic moments \\
\hline \multirow[t]{2}{*}{$\overline{\Delta \Delta p}$} & $\mu_{\mathrm{val}}$ & $\frac{2 \sqrt{2}}{3} \mu_{u}-\frac{2 \sqrt{2}}{3} \mu_{d}$ \\
\hline & $\overline{\mu_{\text {sea }}}$ & $-\frac{2 \sqrt{2}}{3} a\left(1+\alpha^{2}+\frac{\beta^{2}}{3}+\frac{\zeta^{2}}{24}+\frac{17}{16} \gamma^{2}\right) \mu_{u}+\frac{2 \sqrt{2}}{3} a\left(1+\alpha^{2}+\frac{\beta^{2}}{3}+\frac{\zeta^{2}}{24}+\frac{17}{16} \gamma^{2}\right) \mu_{d}$ \\
\hline \multirow[t]{2}{*}{$\Sigma^{*+} \Sigma^{+}$} & $\mu_{\mathrm{val}}$ & $\frac{2 \sqrt{2}}{3} \mu_{u}-\frac{2 \sqrt{2}}{3} \mu_{s}$ \\
\hline & $\overline{\mu_{\text {sea }}}$ & $-\frac{2 \sqrt{2}}{3} a\left(2+\frac{\beta^{2}}{3}+\frac{\zeta^{2}}{24}+\frac{17}{16} \gamma^{2}\right) \mu_{u}-\frac{2 \sqrt{2}}{3} a\left(1-\alpha^{2}\right) \mu_{d}+\frac{2 \sqrt{2}}{3} a\left(\alpha^{2}+\frac{4}{3} \beta^{2}+\frac{\zeta^{2}}{24}+\frac{17}{16} \gamma^{2}\right) \mu_{s}$ \\
\hline \multirow[t]{2}{*}{$\Sigma^{* 0} \Sigma^{0}$} & $\mu_{\mathrm{val}}$ & $\frac{\sqrt{2}}{3} \mu_{u}+\frac{\sqrt{2}}{3} \mu_{d}-\frac{2 \sqrt{2}}{3} \mu_{s}$ \\
\hline & $\mu_{\text {sea }}$ & $-\frac{\sqrt{2}}{3} a\left(3-\alpha^{2}+\frac{\beta^{2}}{3}+\frac{\zeta^{2}}{24}+\frac{17}{16} \gamma^{2}\right) \mu_{u}-\frac{\sqrt{2}}{3} a\left(3-\alpha^{2}+\frac{\beta^{2}}{3}+\frac{\zeta^{2}}{24}+\frac{17}{16} \gamma^{2}\right) \mu_{d}+\frac{2 \sqrt{2}}{3} a\left(\alpha^{2}+\frac{4}{3} \beta^{2}+\frac{\zeta^{2}}{24}+\frac{17}{16} \gamma^{2}\right) \mu_{s}$ \\
\hline \multirow[t]{2}{*}{$\Xi^{* 0} \Xi^{0}$} & $\mu_{\mathrm{val}}$ & $\frac{2 \sqrt{2}}{3} \mu_{u}-\frac{2 \sqrt{2}}{3} \mu_{s}$ \\
\hline & $\mu_{\text {sea }}$ & $-\frac{2 \sqrt{2}}{3} a\left(2+\frac{\beta^{2}}{3}+\frac{\zeta^{2}}{24}+\frac{17}{16} \gamma^{2}\right) \mu_{u}-\frac{2 \sqrt{2}}{3} a\left(1-\alpha^{2}\right) \mu_{d}+\frac{2 \sqrt{2}}{3} a\left(\alpha^{2}+\frac{4}{3} \beta^{2}+\frac{\zeta^{2}}{24}+\frac{17}{16} \gamma^{2}\right) \mu_{s}$ \\
\hline \multirow[t]{2}{*}{$\Sigma^{* 0} \Lambda$} & $\mu_{\mathrm{val}}$ & $\sqrt{\frac{2}{3}} \mu_{u}-\sqrt{\frac{2}{3}} \mu_{d}$ \\
\hline & $\mu_{\text {sea }}$ & $-\sqrt{\frac{2}{3}} a\left(1+\alpha^{2}+\frac{\beta^{2}}{3}+\frac{\zeta^{2}}{24}+\frac{17}{16} \gamma^{2}\right) \mu_{u}+\sqrt{\frac{2}{3}} a\left(1+\alpha^{2}+\frac{\beta^{2}}{3}+\frac{\zeta^{2}}{24}+\frac{17}{16} \gamma^{2}\right) \mu_{d}$ \\
\hline \multirow[t]{2}{*}{$\Sigma_{c}^{*++} \Sigma_{c}^{++}$} & $\mu_{\mathrm{val}}$ & $-\frac{2 \sqrt{2}}{3} \mu_{u}+\frac{2 \sqrt{2}}{3} \mu_{c}$ \\
\hline & $\overline{\mu_{\text {sea }}}$ & $\frac{2 \sqrt{2}}{3} a\left(2+\alpha^{2}+\frac{\beta^{2}}{3}+\frac{\zeta^{2}}{24}+\frac{\gamma^{2}}{16}\right) \mu_{u}+\frac{2 \sqrt{2}}{3} a\left(1-\gamma^{2}\right) \mu_{d}+\frac{2 \sqrt{2}}{3} a\left(\alpha^{2}-\gamma^{2}\right) \mu_{s}-\frac{\sqrt{2}}{12} a\left(3 \zeta^{2}+25 \gamma^{2}\right) \mu_{c}$ \\
\hline \multirow[t]{2}{*}{$\Sigma_{c}^{*+} \Sigma_{c}^{+}$} & $\mu_{\mathrm{val}}$ & $-\frac{\sqrt{2}}{3} \mu_{u}-\frac{\sqrt{2}}{3} \mu_{d}+\frac{2 \sqrt{2}}{3} \mu_{c}$ \\
\hline & $\overline{\mu_{\text {sea }}}$ & $\frac{\sqrt{2}}{3} a\left(2+\alpha^{2}+\frac{\beta^{2}}{3}+\frac{\zeta^{2}}{24}-\frac{15}{16} \gamma^{2}\right) \mu_{u}+\frac{\sqrt{2}}{3} a\left(2+\alpha^{2}+\frac{\beta^{2}}{3}+\frac{\zeta^{2}}{24}-\frac{15}{16} \gamma^{2}\right) \mu_{d}+\frac{2 \sqrt{2}}{3} a\left(\alpha^{2}-\gamma^{2}\right) \mu_{s}-\frac{\sqrt{2}}{12} a\left(3 \zeta^{2}+25 \gamma^{2}\right) \mu_{c}$ \\
\hline \multirow[t]{2}{*}{$\Omega_{c}^{* 0} \Omega_{c}^{0}$} & $\mu_{\mathrm{val}}$ & $-\frac{2 \sqrt{2}}{3} \mu_{s}+\frac{2 \sqrt{2}}{3} \mu_{c}$ \\
\hline & $\overline{\mu_{\text {sea }}}$ & $\frac{2 \sqrt{2}}{3} a\left(\alpha^{2}-\gamma^{2}\right) \mu_{u}+\frac{2 \sqrt{2}}{3} a\left(\alpha^{2}-\gamma^{2}\right) \mu_{d}+\frac{2 \sqrt{2}}{3} a\left(2 \alpha^{2}+\frac{4}{3} \beta^{2}+\frac{\zeta^{2}}{24}+\frac{\gamma^{2}}{16}\right) \mu_{s}-\frac{\sqrt{2}}{12} a\left(3 \zeta^{2}+25 \gamma^{2}\right) \mu_{c}$ \\
\hline \multirow[t]{2}{*}{$\Sigma_{c}^{*+} \Lambda_{c}^{+}$} & $\mu_{\mathrm{val}}$ & $\sqrt{\frac{2}{3}} \mu_{u}-\sqrt{\frac{2}{3}} \mu_{d}$ \\
\hline & $\mu_{\text {sea }}$ & $-\sqrt{\frac{2}{3} a}\left(1+\alpha^{2}+\frac{\beta^{2}}{3}+\frac{\zeta^{2}}{24}+\frac{17}{16} \gamma^{2}\right) \mu_{u}+\sqrt{\frac{2}{3}} a\left(1+\alpha^{2}+\frac{\beta^{2}}{3}+\frac{\zeta^{2}}{24}+\frac{17}{16} \gamma^{2}\right) \mu_{d}$ \\
\hline \multirow[t]{2}{*}{$\Xi_{c}^{*+} \Xi_{c}^{+}$} & $\mu_{\mathrm{val}}$ & $\sqrt{\frac{2}{3}} \mu_{u}-\sqrt{\frac{2}{3}} \mu_{s}$ \\
\hline & $\mu_{\text {sea }}$ & $-\sqrt{\frac{2}{3} a\left(2+\frac{\beta^{2}}{3}+\frac{\zeta^{2}}{24}+\frac{17}{16} \gamma^{2}\right) \mu_{u}-\sqrt{\frac{2}{3}} a\left(1-\alpha^{2}\right) \mu_{d}+\sqrt{\frac{2}{3} a}\left(\alpha^{2}+\frac{4}{3} \beta^{2}+\frac{\zeta^{2}}{24}+\frac{17}{16} \gamma^{2}\right) \mu_{s}}$ \\
\hline \multirow[t]{2}{*}{$\Xi_{c c}^{*++} \Xi_{c c}^{++}$} & $\mu_{\mathrm{val}}$ & $\frac{2 \sqrt{2}}{3} \mu_{u}-\frac{2 \sqrt{2}}{3} \mu_{c}$ \\
\hline & $\overline{\mu_{\text {sea }}}$ & $-\frac{2 \sqrt{2}}{3} a\left(2+\alpha^{2}+\frac{\beta^{2}}{3}+\frac{\zeta^{2}}{24}+\frac{\gamma^{2}}{16}\right) \mu_{u}-\frac{2 \sqrt{2}}{3} a\left(1-\gamma^{2}\right) \mu_{d}-\frac{2 \sqrt{2}}{3} a\left(\alpha^{2}-\gamma^{2}\right) \mu_{s}+\frac{\sqrt{2}}{12} a\left(3 \zeta^{2}+25 \gamma^{2}\right) \mu_{c}$ \\
\hline \multirow[t]{2}{*}{$\overline{\Omega_{c c}^{*+} \Omega_{c c}^{+}}$} & $\mu_{\mathrm{val}}$ & $\frac{2 \sqrt{2}}{3} \mu_{s}-\frac{2 \sqrt{2}}{3} \mu_{c}$ \\
\hline & $\mu_{\text {sea }}$ & $-\frac{2 \sqrt{2}}{3} a\left(\alpha^{2}-\gamma^{2}\right) \mu_{u}-\frac{2 \sqrt{2}}{3} a\left(\alpha^{2}-\gamma^{2}\right) \mu_{d}-\frac{2 \sqrt{2}}{3} a\left(2 \alpha^{2}+\frac{4}{3} \beta^{2}+\frac{\zeta^{2}}{24}+\frac{\gamma^{2}}{16}\right) \mu_{s}+\frac{\sqrt{2}}{12} a\left(3 \zeta^{2}+25 \gamma^{2}\right)$ \\
\hline \multirow[t]{2}{*}{$\overline{\Sigma^{0} \Lambda}$} & $\mu_{\mathrm{val}}$ & $\frac{1}{\sqrt{3}} \mu_{u}-\frac{1}{\sqrt{3}} \mu_{d}$ \\
\hline & $\overline{\mu_{\text {sea }}}$ & $-\frac{a}{\sqrt{3}}\left(1+\alpha^{2}+\frac{\beta^{2}}{3}+\frac{\zeta^{2}}{24}+\frac{17}{16} \gamma^{2}\right) \mu_{u}+\frac{a}{\sqrt{3}}\left(1+\alpha^{2}+\frac{\beta^{2}}{3}+\frac{\zeta^{2}}{24}+\frac{17}{16} \gamma^{2}\right) \mu_{d}$ \\
\hline \multirow[t]{2}{*}{$\Lambda_{c}^{+} \Sigma_{c}^{+}$} & $\mu_{\mathrm{val}}$ & $\frac{1}{\sqrt{3}} \mu_{u}-\frac{1}{\sqrt{3}} \mu_{d}$ \\
\hline & $\mu_{\text {sea }}$ & $-\frac{1}{\sqrt{3}} a\left(1+\alpha^{2}+\frac{\beta^{2}}{3}+\frac{\zeta^{2}}{24}+\frac{17}{16} \gamma^{2}\right) \mu_{u}+\frac{1}{\sqrt{3}} a\left(1+\alpha^{2}+\frac{\beta^{2}}{3}+\frac{\zeta^{2}}{24}+\frac{17}{16} \gamma^{2}\right) \mu_{d}$ \\
\hline \multirow[t]{2}{*}{$\Xi_{c}^{\prime+} \Xi_{c}^{+}$} & $\mu_{\mathrm{val}}$ & $\frac{1}{\sqrt{3}} \mu_{u}-\frac{1}{\sqrt{3}} \mu_{s}$ \\
\hline & $\mu_{\text {sea }}$ & $-\frac{1}{\sqrt{3}} a\left(2+\frac{\beta^{2}}{3}+\frac{\zeta^{2}}{24}+\frac{17}{16} \gamma^{2}\right) \mu_{u}-\frac{1}{\sqrt{3}} a\left(1-\alpha^{2}\right) \mu_{d}+\frac{1}{\sqrt{3}} a\left(\alpha^{2}+\frac{4}{3} \beta^{2}+\frac{\zeta^{2}}{24}+\frac{17}{16} \gamma^{2}\right) \mu_{s}$ \\
\hline
\end{tabular}

TABLE III: Valence and sea contributions of the low lying and charmed spin $\frac{3}{2}^{+} \rightarrow \frac{1}{2}^{+}$and spin $\frac{1}{2}^{+} \rightarrow \frac{1}{2}^{+}$transition magnetic moments in terms of the $\chi \mathrm{CQM}$ parameters. The spin polarizations for the other transitions can be found from isospin symmetry. 


\begin{tabular}{|c|c|c|c|c|c|c|c|c|c|}
\hline Baryon & $\begin{array}{c}\text { Data } \\
{[3]}\end{array}$ & $\begin{array}{c}\text { NRQM } \\
{[4]}\end{array}$ & $\begin{array}{c}\text { Lattice QCD } \\
{[43]}\end{array}$ & $\begin{array}{c}\text { QCDSR [12] } \\
\text { QSSR [13] }\end{array}$ & $\begin{array}{c}\text { LCQSR } \\
{[14]}\end{array}$ & Valence & Sea & Orbital & Total \\
\hline \hline$\mu(p)$ & $2.79 \pm 0.00$ & 3 & 2.793 & $2.82 \pm 0.26$ & $2.7 \pm 0.5$ & 2.90 & -0.58 & 0.48 & 2.80 \\
$\mu(n)$ & $-1.91 \pm 0.00$ & -2 & $-1.59 \pm 0.21$ & $-1.97 \pm 0.15$ & $-1.8 \pm 0.35$ & -1.85 & 0.18 & -0.44 & -2.11 \\
$\mu\left(\Sigma^{+}\right)$ & $2.46 \pm 0.01$ & 2.88 & $2.37 \pm 0.18$ & $2.31 \pm 0.25$ & $2.2 \pm 0.4$ & 2.50 & -0.51 & 0.40 & 2.39 \\
$\mu\left(\Sigma^{0}\right)$ & $\ldots$ & 0.88 & $0.65 \pm 0.06$ & $0.69 \pm 0.07$ & $0.5 \pm 0.10$ & 0.74 & -0.22 & 0.02 & 0.54 \\
$\mu\left(\Sigma^{-}\right)$ & $-1.16 \pm 0.025$ & -1.12 & $-1.07 \pm 0.11$ & $-1.16 \pm 0.10$ & $-0.8 \pm 0.2$ & -1.02 & 0.06 & -0.36 & -1.32 \\
$\mu\left(\Xi^{0}\right)$ & $-1.25 \pm 0.014$ & -1.53 & $-1.17 \pm 0.10$ & $-1.17 \pm 0.10$ & $-1.3 \pm 0.3$ & -1.29 & 0.14 & -0.09 & -1.24 \\
$\mu\left(\Xi^{-}\right)$ & $-0.651 \pm 0.003$ & -0.53 & $-0.51 \pm 0.07$ & $-0.64 \pm 0.06$ & $-0.7 \pm 0.2$ & -0.59 & 0.03 & 0.06 & -0.50 \\
$\Delta C G$ & $0.49 \pm 0.05$ & 0.0 & $\ldots$ & $\ldots$ & $\ldots$ & 0.53 & -0.08 & 0.01 & 0.46 \\
$\mu(\Lambda)$ & $-0.613 \pm 0.004$ & -0.65 & $-0.50 \pm 0.07$ & $-0.56 \pm 0.15$ & $-0.7 \pm 0.2$ & -0.59 & 0.02 & -0.01 & -0.58 \\
\hline$\mu\left(\Sigma_{c}^{++}\right)$ & $\ldots$ & 2.54 & $\ldots$ & $2.1 \pm 0.3$ & $\ldots$ & 2.32 & -0.52 & 0.40 & 2.20 \\
$\mu\left(\Sigma_{c}^{+}\right)$ & $\ldots$ & 0.54 & $\ldots$ & $0.6 \pm 0.1$ & $\ldots$ & 0.51 & -0.23 & 0.02 & 0.30 \\
$\mu\left(\Sigma_{c}^{0}\right)$ & $\ldots$ & -1.46 & $\ldots$ & $-1.6 \pm 0.2$ & $\ldots$ & -1.30 & 0.06 & -0.36 & -1.60 \\
$\mu\left(\Xi_{c}^{\prime}\right)$ & $\ldots$ & 0.77 & $\ldots$ & $\ldots$ & $\ldots$ & 0.78 & -0.21 & 0.19 & 0.76 \\
$\mu\left(\Xi_{c}^{\prime}\right)$ & $\ldots$ & -1.23 & $\ldots$ & $\ldots$ & $\ldots$ & -1.16 & 0.03 & -0.19 & -1.32 \\
$\mu\left(\Omega_{c}^{0}\right)$ & $\ldots$ & -0.99 & $\ldots$ & $\ldots$ & $\ldots$ & -0.93 & 0.04 & -0.01 & -0.90 \\
$\mu\left(\Lambda_{c}^{+}\right)$ & $\ldots$ & 0.39 & $\ldots$ & $0.15 \pm 0.05$ & $0.40 \pm 0.05$ & 0.409 & -0.019 & 0.002 & 0.392 \\
$\mu\left(\Xi_{c}^{+}\right)$ & $\ldots$ & 0.39 & $\ldots$ & $\ldots$ & $0.50 \pm 0.05$ & 0.41 & -0.02 & 0.01 & 0.40 \\
$\mu\left(\Xi_{c}^{0}\right)$ & $\ldots$ & 0.39 & $\ldots$ & $\ldots$ & $0.35 \pm 0.05$ & 0.29 & -0.0003 & -0.01 & 0.28 \\
\hline$\mu\left(\Xi_{c c}^{+}\right)$ & $\ldots$ & -0.15 & $\ldots$ & $\ldots$ & $\ldots$ & -0.025 & 0.111 & -0.080 & 0.006 \\
$\mu\left(\Xi_{c c}^{+}\right)$ & $\ldots$ & 0.85 & $\ldots$ & $\ldots$ & $\ldots$ & 0.79 & -0.02 & 0.07 & 0.84 \\
$\mu\left(\Omega_{c c}^{+}\right)$ & $\ldots$ & 0.73 & $\ldots$ & $\ldots$ & $\ldots$ & 0.706 & -0.013 & 0.004 & 0.697 \\
\hline
\end{tabular}

TABLE IV: Magnetic moment of the low lying and charmed spin $\frac{1}{2}^{+}$baryons with configuration mixing (in units of $\mu_{N}$ ). 


\begin{tabular}{|c|c|c|c|c|c|c|c|c|c|}
\hline Baryon & $\begin{array}{c}\text { Data } \\
{[3]} \\
\end{array}$ & $\begin{array}{c}\text { NRQM } \\
{[4]} \\
\end{array}$ & $\begin{array}{c}\text { Lattice QCD } \\
{[43]}\end{array}$ & $\begin{array}{c}\text { QCDSR } \\
{[12]} \\
\end{array}$ & $\begin{array}{c}\text { LCQSR } \\
{[15]} \\
\end{array}$ & Valence & Sea & Orbital & Total \\
\hline$\mu\left(\Delta^{++}\right)$ & $3.7 \sim 7.5$ & 6 & $4.99 \pm 0.56$ & $4.13 \pm 1.30$ & $4.4 \pm 0.8$ & 4.53 & -0.97 & 0.95 & 4.51 \\
\hline$\mu\left(\Delta^{+}\right)$ & $2.7_{-1.3}^{+1.0} \pm 1.5 \pm 3[44]$ & 3 & $2.49 \pm 0.27$ & $2.07 \pm 0.65$ & $2.2 \pm 0.4$ & 2.27 & -0.61 & 0.34 & 2.00 \\
\hline$\mu\left(\Delta^{0}\right)$ & $\ldots$ & 0.0 & $0.06 \pm 0.0$ & 0.0 & 0.0 & 0.0 & -0.25 & -0.26 & -0.51 \\
\hline$\mu\left(\Delta^{-}\right)$ & $\ldots$ & -3 & $-2.45 \pm 0.27$ & $-2.07 \pm 0.65$ & $-2.2 \pm 0.4$ & -2.27 & 0.12 & -0.87 & -3.02 \\
\hline$\mu\left(\Sigma^{*+}\right)$ & $\ldots$ & 3.35 & $2.55 \pm 0.26$ & $2.13 \pm 0.82$ & $2.7 \pm 0.6$ & 2.74 & -0.67 & 0.62 & 2.69 \\
\hline$\mu\left(\Sigma^{* 0}\right)$ & $\ldots$ & 0.35 & $0.27 \pm 0.05$ & $0.32 \pm 0.15$ & $0.20 \pm 0.05$ & 0.29 & -0.29 & 0.02 & 0.02 \\
\hline$\mu\left(\Sigma^{*-}\right)$ & $\ldots$ & -2.65 & $-2.02 \pm 0.18$ & $-1.66 \pm 0.73$ & $-2.28 \pm 0.5$ & -2.16 & 0.11 & -0.59 & -2.64 \\
\hline$\mu\left(\Xi^{* 0}\right)$ & $\ldots$ & 0.71 & $0.46 \pm 0.07$ & $0.69 \pm 0.29$ & $0.40 \pm 0.08$ & 0.51 & -0.26 & 0.29 & 0.54 \\
\hline$\mu\left(\Xi^{*-}\right)$ & $\ldots$ & -2.29 & $-1.68 \pm 0.12$ & $-1.51 \pm 0.52$ & $-2.0 \pm 0.4$ & -1.64 & 0.08 & -0.31 & -1.87 \\
\hline$\mu\left(\Omega^{-}\right)$ & $\begin{array}{c}-2.02 \pm 0.06 \\
-1.94 \pm 0.31[45]\end{array}$ & -1.94 & $-1.40 \pm 0.10$ & $-1.49 \pm 0.45$ & $-1.65 \pm 0.35$ & -1.76 & 0.08 & -0.03 & -1.71 \\
\hline$\mu\left(\Sigma_{c}^{*++}\right)$ & $\ldots$ & 4.39 & .. & $\ldots$ & $4.81 \pm 1.22$ & 4.09 & -0.80 & 0.63 & 3.92 \\
\hline$\mu\left(\Sigma_{c}^{*+}\right)$ & $\ldots$ & 1.39 & $\ldots$ & $\ldots$ & $2.00 \pm 0.46$ & 1.30 & -0.36 & 0.03 & 0.97 \\
\hline$\mu\left(\Sigma_{c}^{* 0}\right)$ & $\ldots$ & -1.61 & $\ldots$ & $\ldots$ & $-0.81 \pm 0.20$ & -1.50 & 0.09 & -0.58 & -1.99 \\
\hline$\mu\left(\Xi_{c}^{*+}\right)$ & $\ldots$ & 1.74 & $\ldots$ & $\ldots$ & $1.68 \pm 0.42$ & 1.67 & -0.39 & 0.31 & 1.59 \\
\hline$\mu\left(\Xi_{c}^{* 0}\right)$ & $\ldots$ & -1.26 & $\ldots$ & $\ldots$ & $-0.68 \pm 0.18$ & -1.21 & 0.08 & -0.30 & -1.43 \\
\hline$\mu\left(\Omega_{c}^{* 0}\right)$ & $\ldots$ & -0.91 & $\ldots$ & $\ldots$ & $-0.62 \pm 0.18$ & -0.89 & 0.05 & -0.02 & -0.86 \\
\hline$\mu\left(\Xi_{c c}^{*++}\right)$ & $\ldots$ & 2.78 & .. & $\ldots$ & $\ldots$ & 2.78 & -0.44 & 0.32 & 2.66 \\
\hline$\mu\left(\Xi_{c c}^{*+}\right)$ & $\ldots$ & -0.22 & $\cdots$ & $\ldots$ & $\cdots$ & -0.22 & 0.04 & -0.29 & -0.47 \\
\hline$\mu\left(\Omega_{c c}^{*+}\right)$ & $\ldots$ & 0.13 & $\ldots$ & $\ldots$ & $\ldots$ & 0.13 & 0.02 & -0.01 & 0.14 \\
\hline$\mu\left(\Omega_{c c c}^{*++}\right)$ & $\ldots$ & 1.17 & $\ldots$ & $\ldots$ & $\ldots$ & 0.165 & 0.011 & -0.002 & 0.155 \\
\hline
\end{tabular}

TABLE V: The magnetic moments of the low lying and charmed spin $\frac{3}{2}^{+}$baryons (in units of $\mu_{N}$ ). 


\begin{tabular}{|c|c|c|c|c|c|c|c|c|c|}
\hline \multicolumn{10}{|c|}{ Spin $\frac{3}{2}^{+} \rightarrow \frac{1}{2}^{+}$transitions } \\
\hline & Transition & Data & NRQM [4] & Lattice QCD [43] & LCQSR [16] & Valence & Sea & Orbital & Total \\
\hline \multirow[t]{7}{*}{$10 \rightarrow 8$} & $\mu(\Delta p)$ & $3.46 \pm 0.03[46]$ & 2.65 & $2.46 \pm 0.43$ & $2.5 \pm 1.3$ & 2.78 & -0.44 & 0.53 & 2.87 \\
\hline & $\mu\left(\Sigma^{*+} \Sigma^{+}\right)$ & $\ldots$ & 2.42 & $2.61 \pm 0.35$ & $2.1 \pm 0.85$ & 2.38 & -0.41 & 0.29 & 2.26 \\
\hline & $\mu\left(\Sigma^{* 0} \Sigma^{0}\right)$ & $\ldots$ & 1.05 & $1.07 \pm 0.13$ & $0.89 \pm 0.38$ & 1.03 & -0.20 & 0.02 & 0.85 \\
\hline & $\mu\left(\Sigma^{*-} \Sigma^{-}\right)$ & $\ldots$ & -0.32 & $-0.47 \pm 0.09$ & $-0.31 \pm 0.10$ & -0.32 & 0.02 & -0.25 & -0.55 \\
\hline & $\mu\left(\Xi^{* 0} \Xi^{0}\right)$ & $\ldots$ & 2.18 & $-2.77 \pm 0.31$ & $2.2 \pm 0.74$ & 2.24 & -0.39 & 0.27 & 2.12 \\
\hline & $\mu\left(\Xi^{*-} \Xi^{-}\right)$ & $\ldots$ & -0.29 & $0.47 \pm 0.08$ & $-0.31 \pm 0.11$ & -0.26 & 0.02 & -0.23 & -0.47 \\
\hline & $\mu\left(\Sigma^{* 0} \Lambda\right)$ & $\ldots$ & 2.31 & $\ldots$ & $2.3 \pm 1.4$ & 2.42 & -0.39 & 0.47 & 2.50 \\
\hline \multirow[t]{6}{*}{$6 \rightarrow 6$} & $\mu\left(\Sigma_{c}^{*++} \Sigma_{c}^{++}\right)$ & $\ldots$ & -1.51 & $\ldots$ & $-2.8 \pm 1.0$ & -1.45 & 0.38 & -0.30 & -1.37 \\
\hline & $\mu\left(\Sigma_{c}^{*+} \Sigma_{c}^{+}\right)$ & $\ldots$ & -0.11 & $\ldots$ & $-1.2 \pm 0.3$ & -0.101 & 0.110 & -0.012 & -0.003 \\
\hline & $\mu\left(\Sigma_{c}^{* 0} \Sigma_{c}^{0}\right)$ & $\ldots$ & 1.30 & $\ldots$ & $0.5 \pm 0.2$ & 1.25 & -0.04 & 0.27 & 1.48 \\
\hline & $\mu\left(\Xi_{c}^{*+} \Xi_{c}^{\prime}+\right)$ & $\ldots$ & -0.26 & $\ldots$ & $\ldots$ & -0.27 & 0.18 & -0.14 & -0.23 \\
\hline & $\mu\left(\Xi_{c}^{* 0} \Xi_{c}^{\prime 0}\right)$ & $\ldots$ & 1.11 & $\ldots$ & $\ldots$ & 1.14 & -0.04 & 0.14 & 1.24 \\
\hline & $\mu\left(\Omega_{c}^{* 0} \Omega_{c}^{0}\right)$ & $\ldots$ & 0.97 & $\ldots$ & $\ldots$ & 0.98 & -0.03 & 0.01 & 0.96 \\
\hline \multirow[t]{3}{*}{$6 \rightarrow \overline{3}$} & $\mu\left(\Sigma_{c}^{*+} \Lambda_{c}^{+}\right)$ & $\ldots$ & 2.33 & $\ldots$ & $3.8 \pm 1.4$ & 2.30 & -0.37 & 0.47 & 2.40 \\
\hline & $\mu\left(\Xi_{c}^{* 0} \Xi_{c}^{0}\right)$ & $\ldots$ & -0.29 & $\ldots$ & $-0.45 \pm 0.18$ & -0.29 & 0.02 & -0.23 & -0.50 \\
\hline & $\mu\left(\Xi_{c}^{*+} \Xi_{c}^{+}\right)$ & $\ldots$ & 2.14 & $\ldots$ & $4.0 \pm 1.8$ & 2.20 & -0.38 & 0.26 & 2.08 \\
\hline \multirow[t]{3}{*}{$3 \rightarrow 3$} & $\mu\left(\Xi_{c c}^{*++} \Xi_{c c}^{++}\right)$ & $\ldots$ & 1.42 & $\ldots$ & $\ldots$ & 1.42 & -0.37 & 0.28 & 1.33 \\
\hline & $\mu\left(\Xi_{c c}^{*+} \Xi_{c c}^{+}\right)$ & $\ldots$ & -1.22 & $\ldots$ & $\ldots$ & -1.22 & 0.07 & -0.26 & -1.41 \\
\hline & $\mu\left(\Omega_{c c}^{*+} \Omega_{c c}^{+}\right)$ & $\ldots$ & -0.91 & $\ldots$ & $\ldots$ & -0.91 & 0.03 & -0.01 & -0.89 \\
\hline \multicolumn{10}{|c|}{ Spin $\frac{1}{2}^{+} \rightarrow \frac{1}{2}^{+}$transitions } \\
\hline $8 \rightarrow 8$ & $\mu\left(\Sigma^{0} \Lambda\right)$ & $1.61 \pm 0.08[3]$ & 1.52 & $-1.16 \pm 0.15$ & $1.6 \pm 0.3$ & 1.59 & -0.30 & 0.31 & 1.60 \\
\hline \multirow[t]{3}{*}{$\overline{3} \rightarrow 6$} & $\mu\left(\Sigma_{c}^{+} \Lambda_{c}^{+}\right)$ & $\ldots$ & 1.46 & $\ldots$ & $1.5 \pm 0.4$ & 1.51 & -0.24 & 0.29 & 1.56 \\
\hline & $\mu\left(\Xi_{c}^{\prime} 0 \Xi_{c}^{0}\right)$ & $\ldots$ & -0.18 & $\ldots$ & $\ldots$ & -0.18 & 0.01 & -0.14 & -0.31 \\
\hline & $\mu\left(\Xi_{c}^{\prime}+\Xi_{c}^{+}\right)$ & $\ldots$ & 1.33 & $\ldots$ & $\ldots$ & 1.37 & -0.23 & 0.16 & 1.30 \\
\hline
\end{tabular}

TABLE VI: The baryon magnetic moments for the low lying and charmed spin $\frac{3}{2}^{+} \rightarrow \frac{1}{2}^{+}$and $\frac{1}{2}^{+} \rightarrow \frac{1}{2}^{+}$transitions (in units of $\left.\mu_{N}\right)$. 